\title{
Travelling-wave similarity solutions for a steadily translating slender dry patch in a thin fluid film
}

\author{
Y. M. Yatim, ${ }^{1 *}$ B. R. Duffy,${ }^{2 \dagger}$ and S. K. Wilson ${ }^{2 \ddagger}$ \\ ${ }^{1}$ School of Mathematical Sciences, \\ Universiti Sains Malaysia, \\ 11800 Penang, Malaysia \\ ${ }^{2}$ Department of Mathematics and Statistics, \\ University of Strathclyde, \\ Livingstone Tower, 26 Richmond Street, \\ Glasgow G1 1XH, United Kingdom
}

February 10, 2013

\begin{abstract}
A novel family of three-dimensional travelling-wave similarity solutions describing a steadily translating slender dry patch in an infinitely wide thin fluid film on an inclined planar substrate is obtained, the flow being driven by gravity and/or a prescribed constant shear stress on the free surface of the film. For both driving mechanisms, the dry patch has a parabolic shape (which may be concave up or concave down the substrate), and the film thickness increases monotonically away from the contact lines to its uniform far-field value. The two most practically important cases of purely gravity-driven flow and of purely surface-shear-stress-driven flow are analysed separately.
\end{abstract}

Keywords: thin film, lubrication approximation, dry patch, travelling wave, similarity solution

\footnotetext{
* Electronic mail: yazariahmy@usm.my

$\dagger$ Author to whom correspondence should be addressed. Electronic mail: b.r.duffy@strath.ac.uk

$\ddagger$ Electronic mail: s.k.wilson@strath.ac.uk. Presently also a Visiting Fellow in the Oxford Centre for Collaborative Applied Mathematics (OCCAM), University of Oxford, Mathematical Institute, 24-29 St. Giles', Oxford OX1 3LB.
} 


\section{INTRODUCTION}

Finite holes and semi-infinite dry patches can occur in both stationary and flowing fluid films for many different reasons, including dry-out due to localised heating, the presence of air bubbles within the film, inhomogeneities of the substrate, and the presence of surfactants. In particular, there is considerable interest in the occurrence and the non-occurrence of both holes and dry patches in fluid films in a variety of industrial contexts such as heat exchangers and coating processes. Specifically, in heat-transfer devices the presence of dry areas of the substrate must generally be avoided because they typically reduce the efficiency of the heat transfer and can lead to local overheating and possibly corrosion of the substrate, while in coating processes the presence of uncoated regions of the substrate is also generally undesirable because they can seriously degrade the quality of the final product and may make it entirely unusable. As a consequence of these and other practical applications, as well as their inherent interest as fundamental problems in fluid mechanics, the problems of the formation, stability and evolution of holes and dry patches in a fluid film are of enduring theoretical, experimental and practical interest.

The pioneering study of the opening or closing of an axisymmetric hole in a stationary fluid film was performed by Taylor and Michael $^{1}$, who considered an unbounded fluid film on a horizontal substrate subject to gravity and surface-tension effects. In this situation there is a unique energetically unstable equilibrium hole configuration provided that the film is sufficiently thin. Taylor and Michael $^{1}$ conjectured that non-equilibrium holes that are smaller than the equilibrium hole will close while those that are larger will open, and found this prediction to be in good agreement with the results of their own physical experiments involving making holes of various sizes in a layer of mercury. Subsequently, various aspects of the stability and unsteady evolution of both axisymmetric and non-axisymmetric holes in a thin fluid film have been studied by, for example, Moriarty and Schwartz ${ }^{2}$, Wilson and Terrill ${ }^{3}$, López, Miksis, and Bankoff 4 , and Bankoff et al. ${ }^{5}$.

The pioneering study of a semi-infinite dry patch in a flowing fluid film driven either by 
gravity or by a prescribed surface shear stress due to an external air flow was performed by Hartley and Murgatroyd ${ }^{6}$ and extended by Murgatroyd ${ }^{7}$. Hartley and Murgatroyd ${ }^{6}$ considered $^{-}$ two (different) criteria, namely a force-balance criterion based on a balance between surfacetension and pressure forces at the stagnation point at the apex of a dry patch and a minimum energy criterion for a stable fluid film, both of which they used to predict the critical film thickness and flow rate for a dry patch to persist. Early experiments on the shape and structure of a dry patch in a fluid film draining under gravity down the outside of a vertical circular cylinder were performed by Ponter et al. ${ }^{8}$. The shape and structure of a dry patch in a fluid film draining under gravity down an inclined plane has been extensively studied both experimentally and theoretically by Wilson ${ }^{9}$, Podgorski, Flesselles, and Limat ${ }^{10,11}$, Rio, Daerr, and Limat ${ }^{12}$, Rio and Limat ${ }^{13}$, and Sébilleau, Lebon, and Limat ${ }^{14}$. In particular, Podgorski et al. ${ }^{10,11}$ observed the presence of a "capillary ridge" of fluid at the edge of the dry patch, and derived a model for the flow within the ridge and hence the shape of the dry patch similar to that proposed much earlier by Wilson ${ }^{9}$, and Sébilleau et al. ${ }^{14}$ found good agreement between the predictions of an improved version of the model and experimental results for a sufficiently viscous fluid.

Taking a somewhat different approach, Wilson, Duffy, and Davis ${ }^{15}$ obtained two steady similarity solutions for a slender dry patch in a thin fluid film draining under gravity down an inclined plane, one for the case of weak surface tension and one for the case of strong surface tension. In the former case the solution predicts that the dry patch has a parabolic shape and that the transverse profile of the free surface has a monotonically increasing shape; in the latter case the solution predicts that the dry patch has a quartic shape and that the transverse profile of the free surface has an oscillatory shape with a capillary ridge near the contact line. An existence theory for the mathematical problem in the case of weak surface tension was provided by Agarwal and O'Regan ${ }^{16}$. Subsequently Holland, Wilson, and Duffy ${ }^{17}$ obtained four steady similarity solutions for a slender dry patch in a thin fluid film in the presence of thermocapillarity effects. All of these works concern steady dry patches, but recently the present authors have obtained unsteady similarity solutions for an opening or closing slender dry patch with a fixed apex in a thin fluid film driven by a prescribed constant surface shear stress (Yatim, Duffy, and 
Wilson $\left.^{18}\right)$; somewhat unexpectedly, it turns out that there is no corresponding solution for a dry patch in a thin film of either a Newtonian fluid or a non-Newtonian power-law fluid that is driven by gravity (Yatim et al. ${ }^{19,20}$ ). Earlier, Betelú and Diez ${ }^{21}$ obtained a rather different unsteady similarity solution that describes two semi-infinite contact lines that meet to form a "dry line" (rather than a dry patch); the dry line vanishes at a "welding point" which moves at constant velocity.

The contribution of the present work is to obtain and analyse a novel family of threedimensional travelling-wave similarity solution describing a steadily translating slender dry patch in an infinitely wide thin fluid film on an inclined planar substrate, the flow being driven by gravity and/or a prescribed constant shear stress on the free surface of the film. For both driving mechanisms, the dry patch has a parabolic shape (which may be concave up or concave down the substrate), and the film thickness increases monotonically away from the contact lines to its uniform far-field value.

\section{PROBLEM FORMULATION}

\section{A. Unsteady thin-film flow around a steadily translating dry patch}

Consider an infinitely wide thin film of Newtonian fluid with constant density $\rho$ and constant viscosity $\mu$ on a planar substrate inclined at an angle $\alpha$ to the horizontal, subject to gravitational acceleration $g$ and a prescribed constant shear stress on its free surface, acting up or down the slope. We shall be concerned with unsteady three-dimensional flow of such a film around a dry patch that translates steadily up or down the substrate, as sketched in Fig. 1. The inclination angle of the substrate, $\alpha$, is taken to satisfy $0<\alpha<\pi / 2$ or $\pi / 2<\alpha<\pi$, the cases of a horizontal substrate $(\alpha=0$ or $\pi$ ) or a vertical substrate $(\alpha=\pi / 2)$ being excluded at the outset. When $0<\alpha<\pi / 2$ the fluid is on the upper side of the substrate (the sessile case), and when $\pi / 2<\alpha<\pi$ it is on the underside of the substrate (the pendent case).

Cartesian axes Oxyz with the $x$ axis down the line of greatest slope and the $z$ axis normal 


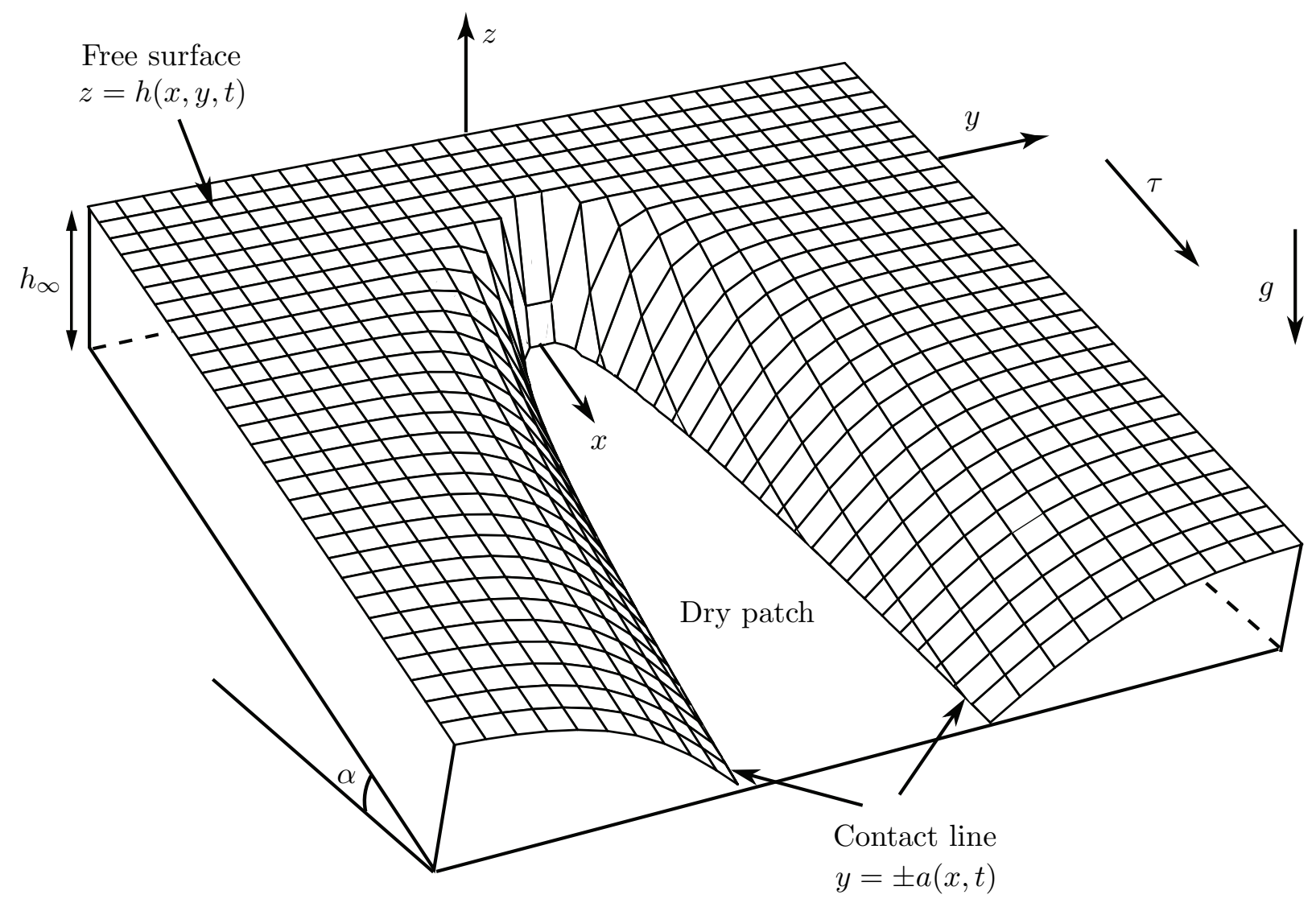

FIG. 1: Sketch of the geometry of the problem: a steadily translating dry patch in a thin fluid film on a planar substrate inclined at an angle $\alpha$ to the horizontal.

to the substrate are adopted, with the substrate at $z=0$; the fluid pressure and velocity are denoted by $p$ and $(u, v, w)$, and the free surface profile of the film is denoted by $z=h(x, y, t)$, where $t$ denotes time. We define the prescribed surface shear stress $\tau$ to be positive (negative) if it acts down (up) the substrate; the case $\tau=0$ corresponds to purely gravity-driven flow.

We are concerned with unsteady flow around a steadily translating dry patch in a film of uniform thickness $h_{\infty}$ at infinity (that is, in a film that would be of uniform thickness $h_{\infty}$ everywhere if the dry patch were absent). We shall restrict attention to dry patches that are symmetric about $y=0$ (so that $h$ is even in $y$ ) with (unknown) semi-width $a=a(x, t)$, so that the fluid occupies $|y| \geq a$, and $h=0$ at the three-phase contact lines $y= \pm a$.

First we non-dimensionalise and scale the variables. It is natural to scale $z$ with the thickness $h_{\infty}$, and to scale $u, p-p_{\text {a }}$ (where $p_{\text {a }}$ denotes the constant atmospheric pressure) and $\tau$ with 
the velocity, pressure and shear-stress scales $U_{\mathrm{S}}=\rho g h_{\infty}^{2} \sin \alpha / \mu, \rho g h_{\infty}|\cos \alpha|$ and $\mu U_{\mathrm{S}} / h_{\infty}$ $\left(=\rho g h_{\infty} \sin \alpha\right)$, respectively, associated with steady unidirectional gravity-driven flow of a film of uniform thickness $h_{\infty}$ down an inclined plane. We take the dry patch to be slender, that is, varying much more slowly in the longitudinal $(x)$ direction than in the transverse $(y)$ direction; specifically, we consider dry patches for which the $x$ and $y$ scales are of orders $\epsilon^{-2} h_{\infty}$ and $\epsilon^{-1} h_{\infty}$, and for which the $v$ and $w$ scales are correspondingly of orders $\epsilon U_{\mathrm{S}}$ and $\epsilon^{2} U_{\mathrm{S}}$, where $\epsilon(\ll 1)$ is a small aspect ratio, namely the ratio of a typical transverse length scale to a typical longitudinal length scale. The time scale associated with the flow is then $h_{\infty} / \epsilon^{2} U_{\mathrm{S}}$. We therefore non-dimensionalise and scale the variables according to

$$
\begin{gathered}
x=\frac{h_{\infty}|\tan \alpha|}{\epsilon^{2}} x^{*}, \quad y=\frac{h_{\infty}}{\epsilon} y^{*}, \quad z=h_{\infty} z^{*}, \quad t=\frac{h_{\infty}|\tan \alpha|}{\epsilon^{2} U_{\mathrm{S}}} t^{*}, \quad h=h_{\infty} h^{*}, \quad a=\frac{h_{\infty}}{\epsilon} a^{*}, \\
u=U_{\mathrm{S}} u^{*}, \quad v=\frac{\epsilon U_{\mathrm{S}}}{|\tan \alpha|} v^{*}, \quad w=\frac{\epsilon^{2} U_{\mathrm{S}}}{|\tan \alpha|} w^{*}, \quad p=p_{\mathrm{a}}+\rho g h_{\infty}|\cos \alpha| p^{*}, \quad \tau=\frac{\mu U_{\mathrm{S}}}{h_{\infty}} \tau^{*},
\end{gathered}
$$

in which the factor $|\tan \alpha|$ arises from the ratio of the longitudinal driving component of gravity, $g \sin \alpha$, to the transverse spreading component $g \cos \alpha$; different values of $\alpha$ correspond merely to different scales in (1), so that the value of $\alpha$ will not appear explicitly in the non-dimensional governing equations and boundary conditions, and the only distinction to be made is between sessile cases $(\cos \alpha>0)$ and pendent cases $(\cos \alpha<0)$. It is then convenient to introduce the notation $S_{g}=\operatorname{sgn}(\cos \alpha)= \pm 1$ to denote the sign of the transverse component of gravity, that is, the $\operatorname{sign}$ of $\cos \alpha$, so that $S_{g}=+1$ in the sessile case and $S_{g}=-1$ in the pendent case.

The flow everywhere is taken to involve a balance between gravity and viscous forces. In particular, we will neglect surface-tension effects in the normal-stress balance at the free surface; this is justified provided that (in dimensional terms) $\gamma\left|h_{y y}\right| \ll \rho g h_{\infty}|\cos \alpha|$, where $\gamma$ denotes the coefficient of surface tension. With the scaling (1) this condition reduces to $\epsilon^{2} \ll B$, where $B=\rho g h_{\infty}^{2}|\cos \alpha| / \gamma$ is an appropriate Bond number; thus surface tension may be neglected provided that the transverse length scale over which the film profile changes is sufficiently large.

With stars on non-dimensional variables dropped for clarity, at leading order in $\epsilon$ the gov- 
erning continuity and Navier-Stokes equations give

$$
\begin{gathered}
u_{x}+v_{y}+w_{z}=0, \\
u_{z z}+1=0, \quad-p_{y}+v_{z z}=0, \quad-p_{z}-S_{g}=0 .
\end{gathered}
$$

The solution of (2) and (3) subject to the boundary conditions of no slip and no penetration on the substrate $z=0$ :

$$
u=v=w=0
$$

leading order balances of normal and tangential stresses on the free surface $z=h$ :

$$
p=0, \quad u_{z}=\tau, \quad v_{z}=0,
$$

and the kinematic condition on $z=h$ :

$$
h_{t}+\bar{u}_{x}+\bar{v}_{y}=0
$$

where the local fluxes $\bar{u}=\bar{u}(x, y, t)$ and $\bar{v}=\bar{v}(x, y, t)$ are defined by

$$
\bar{u}=\int_{0}^{h} u \mathrm{~d} z, \quad \bar{v}=\int_{0}^{h} v \mathrm{~d} z,
$$

is simply

$$
\begin{gathered}
p=S_{g}(h-z), \\
u=\frac{1}{2}(2 h-z) z+\tau z, \\
v=-\frac{S_{g} h_{y}}{2}(2 h-z) z, \\
w=\frac{S_{g}}{2}\left(h h_{y y}+h_{y}^{2}-\frac{h_{y y} z}{3}\right) z^{2}-\frac{h_{x} z^{2}}{2} .
\end{gathered}
$$

Substituting (9) and (10) into (7) gives

$$
\bar{u}=\frac{h^{3}}{3}+\frac{\tau h^{2}}{2}, \quad \bar{v}=-\frac{S_{g} h^{3} h_{y}}{3}
$$

and hence (6) yields the governing partial differential equation for $h$ :

$$
h_{t}=\frac{S_{g}}{3}\left(h^{3} h_{y}\right)_{y}-\frac{1}{3}\left(h^{3}\right)_{x}-\frac{\tau}{2}\left(h^{2}\right)_{x} .
$$

Once $h$ is determined from (13) the solution for $p, u, v$ and $w$ in (8)-(11) is known. 
From (12) we have $\bar{u}=0$ at $y= \pm a$, so that the zero-mass-flux condition at the contact lines, namely

$$
\bar{v}= \pm a_{x} \bar{u} \quad \text { at } \quad y= \pm a
$$

reduces to $\bar{v}=0$ at $y= \pm a$, and therefore the contact-line conditions are

$$
h=0 \quad \text { at } \quad y= \pm a, \quad h^{3} h_{y} \rightarrow 0 \quad \text { as } \quad y \rightarrow \pm a .
$$

Far from the dry patch the film is of unit thickness, that is,

$$
h \rightarrow 1 \quad \text { as }|y| \rightarrow \infty
$$

and the solution (8)-(11) takes the form $p=p_{\infty}(z), u=u_{\infty}(z), v \equiv 0$ and $w \equiv 0$, where

$$
p_{\infty}=S_{g}(1-z), \quad u_{\infty}=\frac{1}{2}(2-z) z+\tau z,
$$

representing steady unidirectional flow up or down the substrate. The depth-averaged velocity $U_{\infty} \mathbf{i}$ associated with the flow (17) and the shear stress acting on the substrate due to this flow, namely $\tau_{\infty}=\mathrm{d} u_{\infty} / \mathrm{d} z$ at $z=0$, are given by

$$
U_{\infty}=\frac{1}{3}+\frac{\tau}{2}, \quad \tau_{\infty}=1+\tau
$$

these quantities will reappear when we analyse the solutions for dry patches. It transpires that in the case $\tau=-1$ (i.e. $\tau_{\infty}=0$ ) there is no solution of (13), (15) and (16) of the general form that we shall be considering, and so for brevity we exclude this case at the outset. Thus although the velocity $U_{\infty}$ may be positive, negative or zero, the stress $\tau_{\infty}$ may be positive or negative but not zero; it is then convenient to introduce the notation $S_{\infty}=\operatorname{sgn}\left(\tau_{\infty}\right)= \pm 1$ to denote the sign of $\tau_{\infty}$.

\section{B. A travelling-wave similarity solution}

Equation (13) has a travelling-wave similarity solution, describing a steadily translating dry patch, of the form

$$
\left.\begin{array}{ll}
h=F(\eta), \quad \eta=\frac{y}{\sqrt{S_{\infty} S_{g}(x-c t)}} & \text { if } \quad S_{\infty} S_{g}(x-c t) \geq 0, \\
h=1 & \text { if } S_{\infty} S_{g}(x-c t)<0,
\end{array}\right\}
$$


where the function $F=F(\eta)(\geq 0)$ of the similarity variable $\eta$ represents the cross-sectional profile of the fluid film, and $c$ i (with $c$ positive, negative or zero) is the constant velocity of the dry patch up or down the substrate; both $F$ and $c$ are to be determined. Equation $(19)_{1}$ represents the dry patch in the region where $S_{\infty} S_{g}(x-c t) \geq 0$, and $(19)_{2}$ represents a film of unit thickness in the region where $S_{\infty} S_{g}(x-c t)<0$, which is either ahead of or behind the dry patch, reflecting the fact that either the dry patch will be below its apex, and the film above the apex will be of uniform thickness (as sketched in Fig. 1), or vice versa.

As will be seen shortly, the factor $S_{\infty} S_{g}$ in (19) is introduced to ensure consistency with the asymptotic behaviour of the solution for $h$ near to the dry patch and far from it. Along the line $x=c t$ (on which $|\eta| \rightarrow \infty$ ) the thickness $h$ of the film and its derivatives $h_{y}$ and $h_{y y}$ are continuous (so that $u, v, w$ and $p$ are continuous there), except at the apex of the dry patch at the singular point $x=c t, y=0$, at which the free surface is normal to the substrate, occupying $0 \leq z \leq 1$

In a frame of reference moving with the dry patch (that is, steadily translating with velocity $c \mathbf{i})$, the flow appears steady, with the dry patch stationary. Denoting quantities in this moving frame by upper-case letters we have

$$
X=x-c t, \quad Y=y, \quad Z=z, \quad T=t, \quad H=h, \quad(U, V, W)=(u-c, v, w),
$$

and the similarity solution again takes the form (19) written in terms of $H$, with $\eta$ now given by $\eta=Y / \sqrt{S_{\infty} S_{g} X}$.

With $(19)_{1}$ equation (13) reduces to a nonlinear ordinary differential equation for the crosssectional profile $F(\eta)$, namely

$$
\left(F^{4}\right)^{\prime \prime}+6 S_{\infty} \eta\left(F^{2}+\tau F-c\right) F^{\prime}=0
$$

where a dash denotes differentiation with respect to $\eta$.

We denote the position in $\eta \geq 0$ where $F=0$ (corresponding to the contact-line position $y=a$ ) by $\eta=\eta_{0}$, a scaled measure of the semi-width of the dry patch. Then the fluid lies in 
$|\eta| \geq \eta_{0}$, and

$$
a=\sqrt{S_{\infty} S_{g}(x-c t)} \eta_{0}, \quad \frac{y}{a}=\frac{\eta}{\eta_{0}}
$$

showing that the edge of the dry patch always has a parabolic shape. In fact, with $(19)_{1}$ the level sets of $h$ are the curves $\eta=$ constant, that is, the film thickness is the same at all points $(x, y)$ on each of the parabolae $y^{2} \propto S_{\infty} S_{g}(x-c t)(\geq 0)$.

From (15) we have

$$
F=0 \quad \text { at } \quad \eta=\eta_{0}, \quad F^{3} F^{\prime} \rightarrow 0 \quad \text { as } \quad \eta \rightarrow \eta_{0}
$$

in addition, $F$ must satisfy the far-field condition

$$
F \rightarrow 1 \text { as } \eta \rightarrow \infty
$$

As we shall see, the value of $\eta_{0}$ is not determined as part of the solution, in general, so that (19) represents a one-parameter family of possible similarity solutions.

To understand the nature of the dry-patch solutions (19) we investigate the properties of solutions of (21), (23) and (24); in section III we derive analytical results that provide valuable information about the motion of the dry patch, and which guide the numerical study described in subsection IV A.

\section{PROPERTIES OF THE SIMILARITY SOLUTION}

\section{A. Behaviour near $\eta=\eta_{0}$}

Near the contact line $\eta=\eta_{0}$ it is found from (21) that the profile of the fluid film, $F$, satisfies either

$$
F \sim\left[\frac{9 \eta_{0} S_{\infty} c\left(\eta-\eta_{0}\right)}{2}\right]^{\frac{1}{3}} \text { for } \quad S_{\infty} c>0
$$

or, in the special case of a stationary dry patch, $c=0$,

$$
F \sim\left[-\frac{3 \eta_{0} \tau\left(\eta-\eta_{0}\right)}{2}\right]^{\frac{1}{2}} \text { for } \quad-1<\tau<0 .
$$


In particular, equation (25) shows that if the prescribed surface shear stress $\tau$ either acts downwards $(\tau \geq 0)$ or acts upwards but is sufficiently weak (specifically, if $-1<\tau \leq 0$ ) then the dry patch moves downwards $(c \geq 0)$, whereas if the surface shear stress acts upwards and is sufficiently strong (specifically, if $\tau<-1$ ) then the dry patch moves upwards $(c<0)$.

Equations (25) and (26) show that the free surface has infinite slope at the contact line $\eta=\eta_{0}$, corresponding to a contact angle of $\pi / 2$; thus the lubrication approximation fails there, and there is no freedom to impose additional conditions such as prescribed contact angles. In practice, surface-tension effects (which are neglected in the bulk of the flow) will be important close to the contact line, and, in principle, some additional microscopic physics needs to be introduced into the model in order to remove the well-known contact-line paradox (see, for example, the recent review articles on moving contact lines by Bonn et al. ${ }^{22}$ and Snoeijer and Andreotti $\left.{ }^{23}\right)$. However, as in the famous similarity solution of $\mathrm{Smith}^{24}$ for the spreading of a large drop, in the present problem the flow is dominated by the behaviour in the bulk (namely a balance between viscous and gravity forces) and so it is not necessary to analyse the details of the flow near the contact line in order to determine the macroscopic behaviour.

\section{B. Behaviour in the limit $\eta \rightarrow \infty$}

If $F$ were to have a stationary point in $\eta \geq \eta_{0}$ then (21) would require all derivatives of $F$ to be zero there, indicating that, in fact, $F$ has no stationary points. Thus $F$ (and hence the thickness of the fluid film) increases monotonically with $\eta$ from $F=0$ at $\eta=\eta_{0}$ to $F \rightarrow 1$ as

$\eta \rightarrow \infty$, and so satisfies $0 \leq F<1$. With this result it may be shown that the solution $F$ of (21) has the far-field behaviour (24) only if $S_{\infty}\left(\tau_{\infty}-c\right) \geq 0$, and then we find that

$$
F-1 \propto \frac{1}{\eta} \exp \left(-\frac{3}{4} S_{\infty}\left(\tau_{\infty}-c\right) \eta^{2}\right) \quad \text { as } \quad \eta \rightarrow \infty
$$

if $S_{\infty}\left(\tau_{\infty}-c\right)>0$, and

$$
F \sim 1-\frac{2}{(2+\tau) \eta^{2}} \quad \text { as } \quad \eta \rightarrow \infty
$$

if $c=\tau_{\infty}$, the latter only if $-2<\tau<-1$. 


\section{Physical forms of the dry patch}

The conditions $S_{\infty} c \geq 0$ and $S_{\infty}\left(\tau_{\infty}-c\right) \geq 0$ required for the validity of $(25)-(28)$ show that the velocity of the dry patch, $c$, always satisfies

$$
\text { either } \quad 0 \leq c \leq \tau_{\infty} \quad \text { or } \quad \tau_{\infty} \leq c \leq 0
$$

This result and the fact that the dry patch is situated in the region where $S_{\infty} S_{g}(x-c t) \geq 0$ together provide useful information for determining the possible physical forms of the dry patch; in particular, we deduce immediately that in the sessile case $\left(\cos \alpha>0, S_{g}=+1\right)$ if $\tau_{\infty}>0$ then the dry patch is situated in $x \geq c t$, moves down the substrate $(c \geq 0)$, and has semi-width $a=\eta_{0} \sqrt{x-c t}$, widening with increasing $x$, the fluid film in $x<c t$ being of unit thickness, whereas if $\tau_{\infty}<0$ then the dry patch is situated in $x \leq c t$, moves up the substrate $(c \leq 0)$, and has semi-width $a=\eta_{0} \sqrt{c t-x}$, narrowing with increasing $x$, the fluid film in $x>c t$ being of unit thickness; analogous conclusions may be drawn in the pendent case $\left(\cos \alpha<0, S_{g}=-1\right)$. The physical forms of the dry patch in these various cases are sketched in Fig. 2, in which (a) and (b) show sessile cases, and (c) and (d) show pendent cases; in (a) and (c), with $\tau_{\infty}>0$, the dry patch is moving down the substrate $(c>0)$, whereas in (b) and (d), with $\tau_{\infty}<0$, it is moving up the substrate $(c<0)$.

Other things being equal, at any given instant $t$ the free-surface profile in a sessile (pendent) case on a substrate inclined at an angle $\alpha$ is the mirror image in the plane $x=c t$ of the free-surface profile in a pendent (sessile) case on a substrate inclined at an angle $\pi-\alpha$.

In the Appendix it is shown from the differential equation (21) that the velocity of the dry patch, $c$, may be expressed in terms of integrals of $F$ as

$$
c=\frac{2 I_{3}+3 \tau I_{2}}{6 I_{1}}
$$

where we have defined the constants $I_{n}(n=1,2,3)$ by

$$
I_{n}=\eta_{0}+\int_{\eta_{0}}^{\infty}\left(1-F^{n}\right) \mathrm{d} \eta
$$

we then use (30) to derive additional restrictions on $c$ (beyond (29)) that were useful in determining the structure of the solutions numerically, as described later in subsection IV A. 

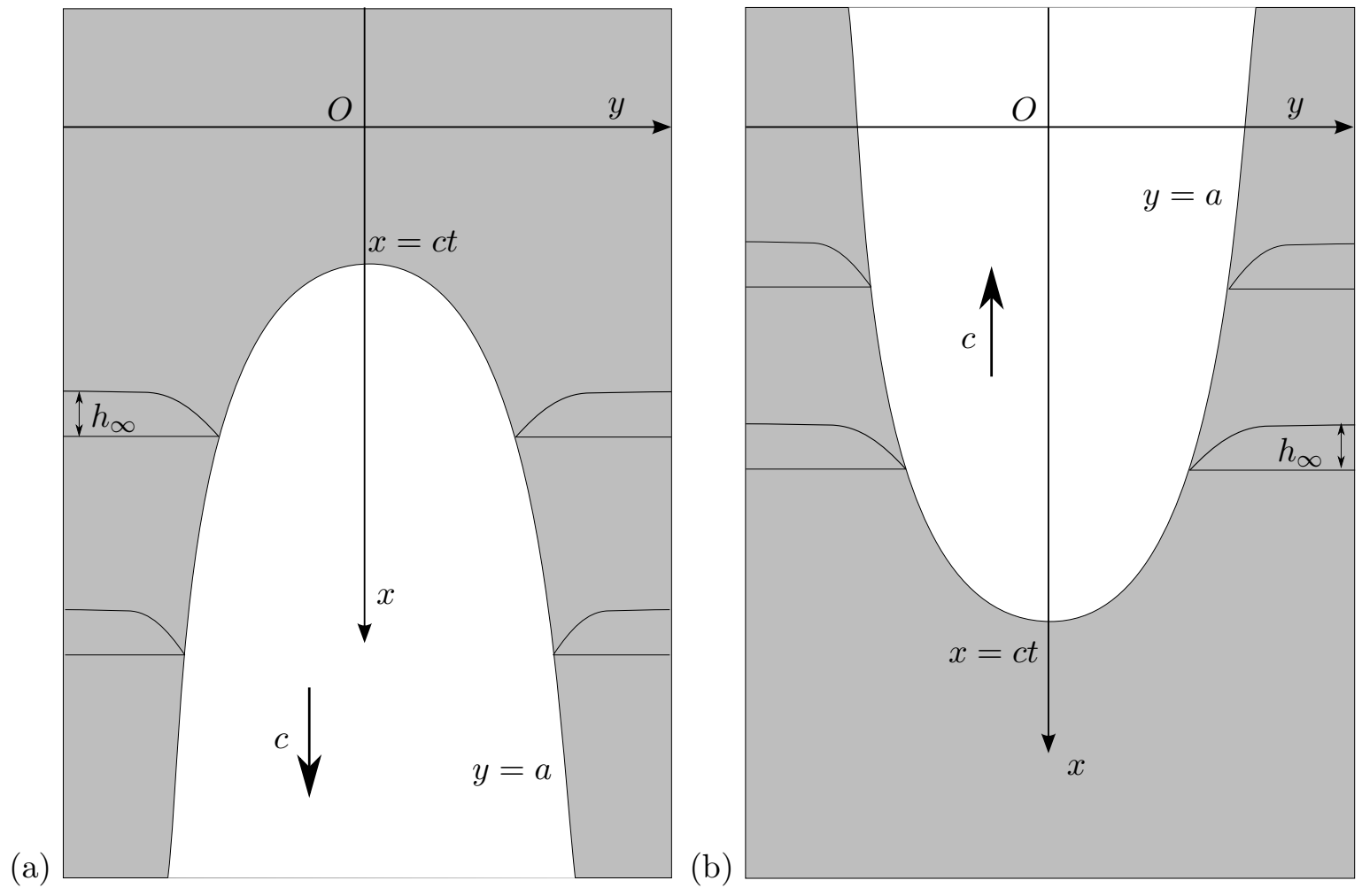

(b)
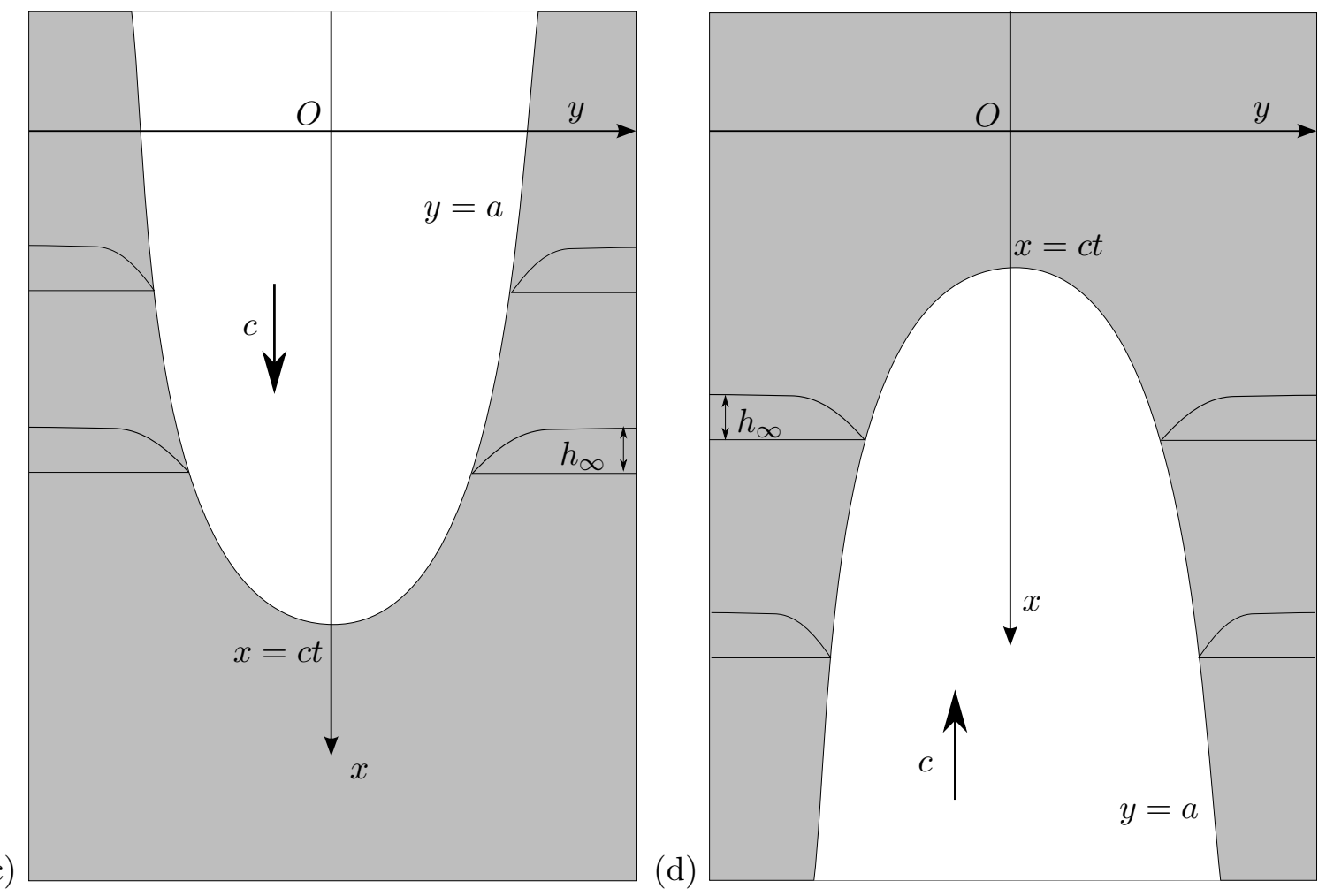

FIG. 2: Sketch of the possible physical forms of the steadily translating dry patch (shown unshaded) in a thin film (shown shaded): (a) and (b) show sessile cases, and (c) and (d) show pendent cases; in (a) and (c), with $\tau_{\infty}>0$, the dry patch is moving down the substrate, and in (b) and (d), with $\tau_{\infty}<0$, it is moving up the substrate. 


\section{Depth-integrated flow}

Analogous to (7) we define local fluxes $\bar{U}$ and $\bar{V}$ in a frame of reference moving with the dry patch by

$$
\bar{U}=\int_{0}^{H} U \mathrm{~d} Z=\bar{u}-c h, \quad \bar{V}=\int_{0}^{H} V \mathrm{~d} Z=\bar{v} .
$$

Then, since $H_{T}=0$ in this frame of reference, the kinematic condition on the free surface takes the steady form

$$
\bar{U}_{X}+\bar{V}_{Y}=0,
$$

so that, although the fluid flow (with velocity $(u, v, w)$ ) is fully three-dimensional, the depthintegrated velocity $(\bar{U}, \bar{V})$ is analogous to a two-dimensional velocity field of an incompressible fluid, for which we may define an effective stream function $\Psi$ by

$$
\bar{U}=\frac{\partial \Psi}{\partial Y}, \quad \bar{V}=-\frac{\partial \Psi}{\partial X}, \quad \Psi=0 \quad \text { at } \quad \eta= \pm \eta_{0} .
$$

Integrating (34) using (12), (21) and (23) we find that

$$
\Psi= \begin{cases}\sqrt{S_{\infty} S_{g} X}\left\{\frac{2 S_{\infty} F^{3} F^{\prime}}{3}+\eta\left(\frac{F^{3}}{3}+\frac{\tau F^{2}}{2}-c F\right)\right\} & \text { if } S_{\infty} S_{g} X \geq 0, \\ \left(U_{\infty}-c\right) Y & \text { if } S_{\infty} S_{g} X<0,\end{cases}
$$

with $U_{\infty}$ again as in (18). Curves of constant $\Psi$ in the $X-Y$ plane may be regarded as effective streamlines of this depth-integrated flow, with the streamline $\Psi=0$ corresponding to the axis $Y=0$ in $S_{\infty} S_{g} X \leq 0$ and to the contact line $Y= \pm a$ in $S_{\infty} S_{g} X \geq 0$. Also $\Psi$ in (35) 1 satisfies $\Psi \sim\left(U_{\infty}-c\right) Y$ in the limit $\eta \rightarrow \pm \infty$, showing that the streamlines are smooth at $X=0$ (that is, at $x=c t$ ), and that the depth-integrated flow is uniform far from the dry patch, with streamlines parallel to the $X$ axis.

Since $h_{y}$ has the same sign as $y$, from $(12)$ we have $\operatorname{sgn}(\bar{V})=\operatorname{sgn}(\bar{v})=-S_{g} \operatorname{sgn}(y)$, showing that the direction of the depth-integrated flow is inwards towards $y=0$ in the sessile case $S_{g}=+1$ but is outwards away from $y=0$ in the pendent case $S_{g}=-1$. Therefore the depthintegrated flow in the moving frame of reference is upward in parts (a) and (c) of Fig. 2, but is downward in parts (b) and (d). 


\section{E. Cross-sectional area and volume flux}

The film is of infinite transverse extent, and its cross-sectional area (at constant $x$ ) is infinite, as is the volume flux of fluid down the substrate. However, the difference between the crosssectional area of the part of the film of unit thickness in the region where $S_{\infty} S_{g}(x-c t)<0$ and that of the part of the film in the region where $S_{\infty} S_{g}(x-c t) \geq 0$ containing the dry patch, denoted $\triangle A$, is finite and is given by

$$
\triangle A=\lim _{y_{\infty} \rightarrow \infty} 2\left(y_{\infty}-\int_{a}^{y_{\infty}} h \mathrm{~d} y\right),
$$

leading to

$$
\triangle A=2 \sqrt{S_{\infty} S_{g}(x-c t)} q_{\text {area }}, \quad \text { where } \quad q_{\text {area }}=I_{1}
$$

for $S_{\infty} S_{g}(x-c t) \geq 0$, where the constants $I_{n}$ are as defined in (31). Similarly, the difference in volume flux in the two parts of the film, denoted $\triangle Q$, is finite and is given by

$$
\triangle Q=\lim _{y_{\infty} \rightarrow \infty} 2\left(U_{\infty} y_{\infty}-\int_{a}^{y_{\infty}} \bar{u} \mathrm{~d} y\right),
$$

leading to

$$
\triangle Q=2 \sqrt{S_{\infty} S_{g}(x-c t)} q_{\text {flux }}, \quad \text { where } \quad q_{\text {flux }}=\frac{I_{3}}{3}+\frac{\tau I_{2}}{2}
$$

for $S_{\infty} S_{g}(x-c t) \geq 0$. Then (30), (37) and (39) show that $q_{\text {flux }}, q_{\text {area }}, \triangle Q$ and $\triangle A$ are related simply by

$$
q_{\text {flux }}=c q_{\text {area }}, \quad \triangle Q=c \triangle A .
$$

\section{DESCRIPTION OF THE SIMILARITY SOLUTIONS}

\section{A. Solutions for $F(\eta)$ and $c$}

A closed-form solution of (21) for the cross-sectional profile $F(\eta)$ is not available, and so it was solved numerically, by means of a shooting method, shooting from a chosen value of the contact-line position $\eta=\eta_{0}$, with chosen values of the surface shear stress $\tau$ and the velocity of 
the dry patch, $c$. The solution $F$ was monitored to see if it satisfied an approximated version of (24), namely

$$
F=1 \quad \text { at } \quad \eta=\eta_{\infty}
$$

where $\eta_{\infty}\left(\gg \eta_{0}\right)$ is a chosen large value of $\eta$, to within a prescribed tolerance; if not then the value of $c$ was changed and the calculation repeated until a solution satisfying (41) for the chosen values of $\eta_{0}, \tau$ and $\eta_{\infty}$ was found. In fact, the numerical computation cannot be started exactly at $\eta=\eta_{0}$ (because of the singular slope there, given by (25)), and so instead it was started from a position $\eta=\eta_{0}+\delta$, where $\delta(>0)$ is small; thus we solved (21) subject to the approximated boundary conditions

$$
F\left(\eta_{0}+\delta\right)=\left(\frac{9 \eta_{0} S_{\infty} c \delta}{2}\right)^{\frac{1}{3}}, \quad F^{\prime}\left(\eta_{0}+\delta\right)=\left(\frac{\eta_{0} S_{\infty} c}{6 \delta^{2}}\right)^{\frac{1}{3}}
$$

obtained from (25). This procedure was then repeated with smaller values of $\delta$ (as small as $\delta=10^{-10}$ ) and larger values of $\eta_{\infty}$ (as large as $\eta_{\infty}=10^{3}$ ) until the solution converged to within a prescribed tolerance.

The main results of the present work concern the dependence of the velocity of the dry patch, $c$, on the position of the contact line, $\eta_{0}$, and the surface shear stress $\tau$. Figure 3 shows plots of $c$ as a function of $\eta_{0}$ obtained by the above procedure, for various values of $\tau$. Each of the solution curves shown in the figure extends over all values of $\eta_{0}(\geq 0)$, but for each $\tau$ (that is, on any particular curve) there is only a finite interval of $c$ values; in particular, $c$ takes a finite nonzero value in the limit of a narrow dry patch, $\eta_{0} \rightarrow 0$, and approaches the constant value $c=U_{\infty}$ in the limit of a wide dry patch, $\eta_{0} \rightarrow \infty$, consistent with the later discussions in subsections IV C and IV D. The curves in Figure 3 are, in general, non-monotonic, so that although for a given semi-width $\eta_{0}$ the velocity $c$ is determined uniquely, for a given velocity $c$ there may be more than one associated semi-width $\eta_{0}$, that is, there may be more than one dry patch with a given velocity. Moreover, curves for different $\tau$ do not intersect each other, showing that the corresponding solutions are distinct; in particular, two dry patches of the same semi-width $\eta_{0}$ with different values of $\tau$ will have different velocities $c$, and two dry patches moving at the same velocity for different values of $\tau$ must have different semi-widths. 


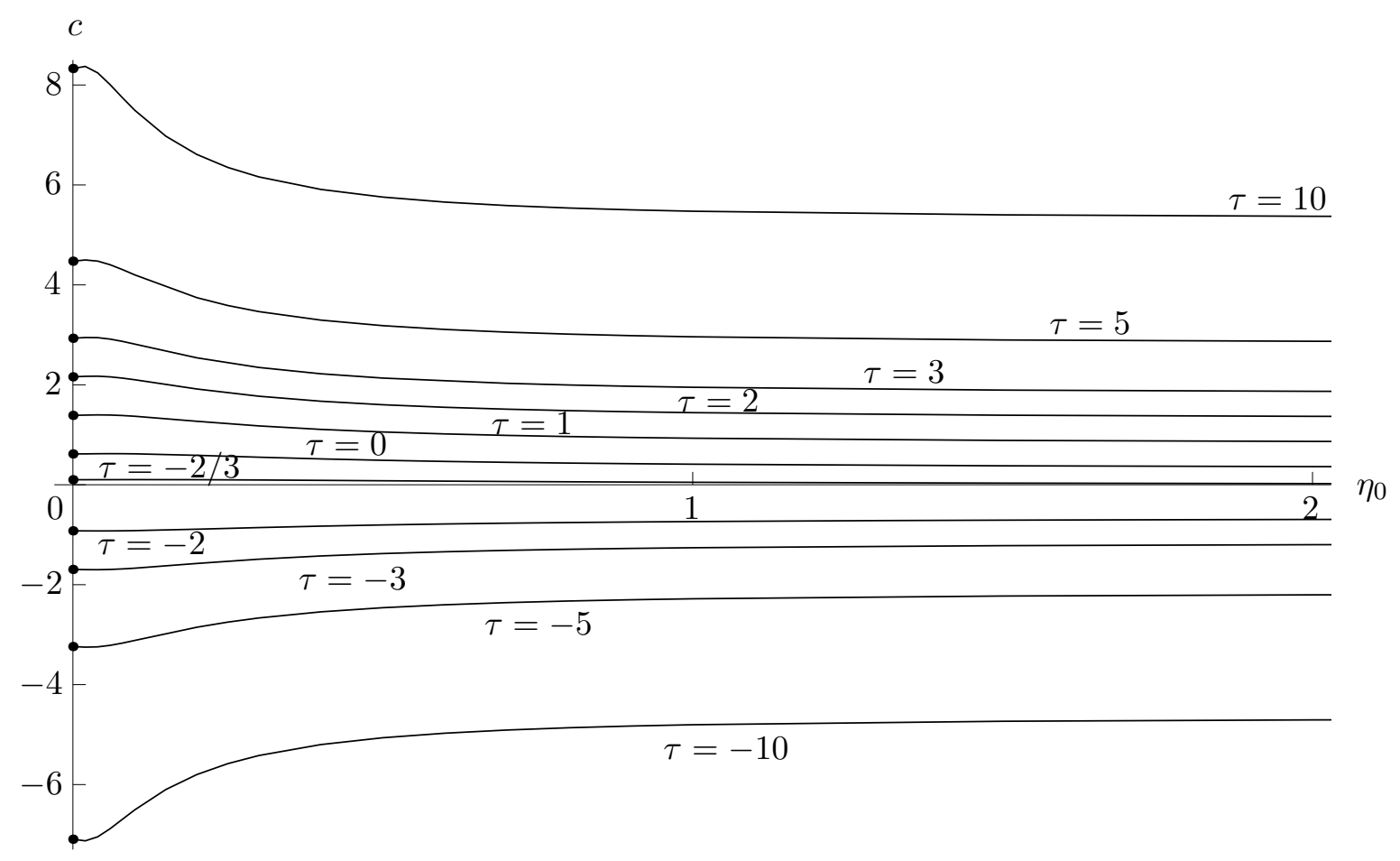

FIG. 3: Plots of $c$ as a function of $\eta_{0}$ for various values of $\tau$.

The apparent simplicity of Fig. 3 is deceptive. Figure 4 shows an enlargement of Fig. 3 (with additional solution curves) near the $\eta_{0}$ axis; all the curves in Fig. 4 are for $\tau<0$, corresponding to an upward surface shear stress. Notable features in Fig. 4, not shown in Fig. 3, are that some (but not all) solution curves in $c \geq 0$ terminate on the $\eta_{0}$ axis (so that for these values of $\tau$ there is a maximum possible semi-width $\eta_{0}$ ), that there is a region below the $\eta_{0}$ axis and above a bounding curve $c=1+\tau<0$ (shown dashed) in which there is no solution (so that, in particular, there is no dry patch with velocity satisfying $-1 / 3 \leq c<0)$, and that some curves in $c<-1 / 3$ (for which $\tau<-4 / 3$ ) lie entirely to the right of this bounding curve (so that for these values of $\tau$ there is a minimum possible semi-width $\eta_{0}$ ). The bounding curve $c=1+\tau$ (on which $c \rightarrow-1 / 3$ as $\eta_{0} \rightarrow \infty$ ) corresponds to one of the two extreme values allowed by the inequality $(29)_{2}$, showing that any solution curve in Fig. 4 that intersects this bounding curve always lies above the point of intersection.

Fig. 5 shows an enlargement of Fig. 4 (that is, a further enlargement of Fig. 3, again with additional solution curves), clarifying the structure of the solution curves for $c \geq 0$ near a critical point in the behaviour, on the $\eta_{0}$ axis. It is found that there are solutions with $c \geq 0$ 


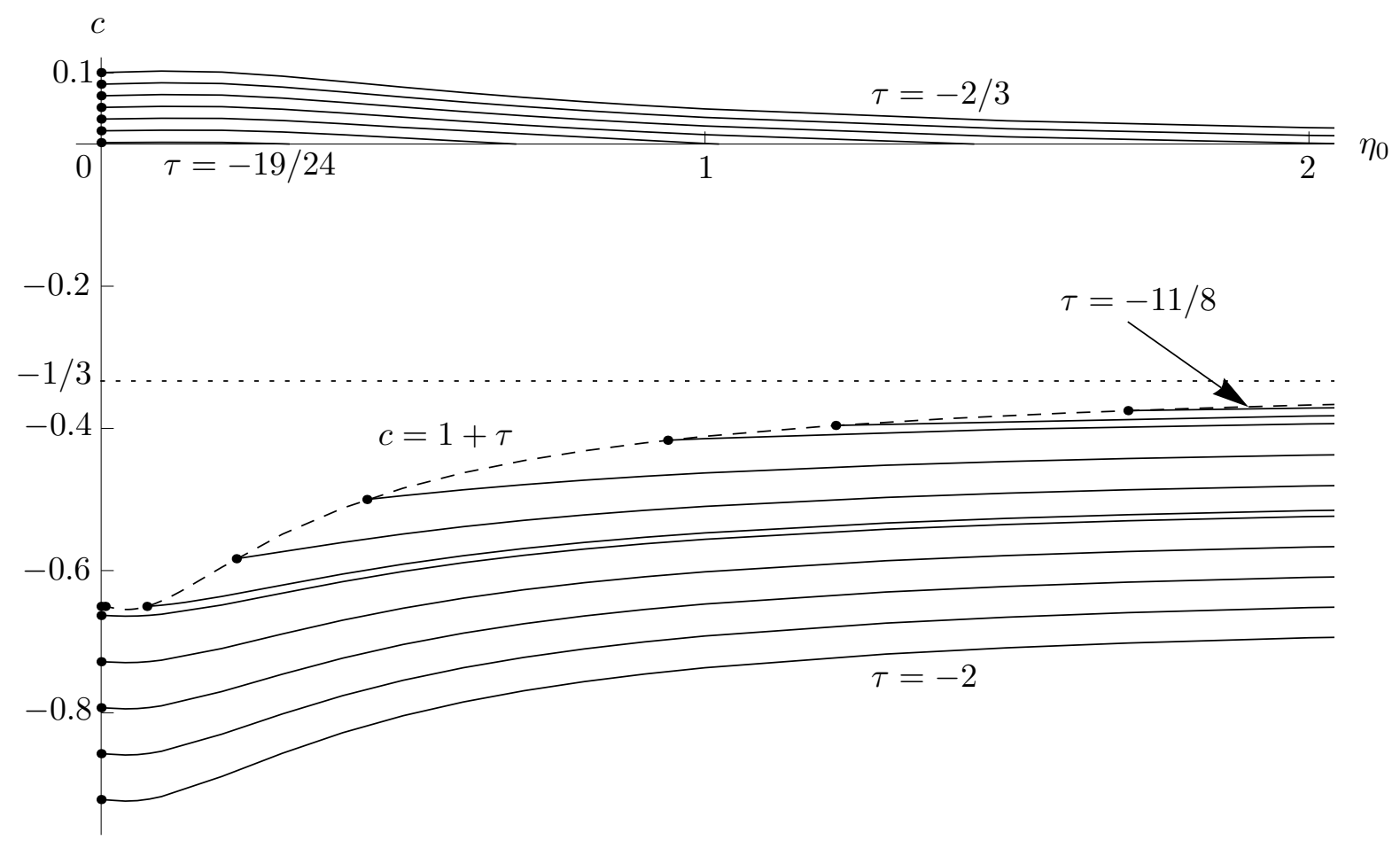

FIG. 4: Enlargement of Fig. 3, showing $c$ as a function of $\eta_{0}$ for $\tau=-2 / 3,-11 / 16,-17 / 24,-35 / 48,-3 / 4$, $-37 / 48,-19 / 24$ (upper group) and $\tau=-11 / 8,-67 / 48,-17 / 12,-3 / 2,-19 / 12, \tau_{\mathrm{c} 4} \simeq-1.6504,-5 / 3,-7 / 4$, $-11 / 6,-23 / 12,-2$ (lower group). The "bounding curve" $c=1+\tau$ is shown with a dashed line, and the asymptote of this curve at large $\eta_{0}$, namely $c=-1 / 3$, is shown with a dotted line.

only for $\tau \geq \tau_{\mathrm{c} 1}$, where the critical value $\tau_{\mathrm{c} 1}$ is given by $\tau_{\mathrm{c} 1} \simeq-0.7947$. The solution curve that passes through $\eta=0, c=0$ corresponds to a second critical value $\tau=\tau_{\mathrm{c} 2}$, where $\tau \simeq-0.7941$; solutions for $\tau_{\mathrm{c} 1} \leq \tau<\tau_{\mathrm{c} 2}$ have not only a maximum possible width but also a (nonzero) minimum possible width. Moreover, the dry patch can be stationary (that is, $c=0$ ) only if $\tau$ satisfies $\tau_{\mathrm{c} 1} \leq \tau<-2 / 3$, and then for only one particular value of $\eta_{0}$ if either $\tau=\tau_{\mathrm{c} 1}$ or $\tau_{\mathrm{c} 2} \leq \tau<-2 / 3$, or for two particular values of $\eta_{0}$ if $\tau_{\mathrm{c} 1}<\tau \leq \tau_{\mathrm{c} 2}$; if $\tau$ satisfies $\tau \geq-2 / 3$ or $\tau<\tau_{\mathrm{c} 1}$ then the dry patch cannot be stationary. Correspondingly, for a prescribed value of $\eta_{0}$ the dry patch can be stationary for only one particular value of $\tau$. In the special case $\tau=\tau_{\mathrm{c} 1}$ there is a single (stationary) solution $c=0$, for which $\eta_{0} \simeq 0.1386$.

Fig. 6 shows another enlargement of Fig. 4 (that is, another further enlargement of Fig. 3, again with additional solution curves), clarifying the structure of the solution curves for $c<0$; again the bounding curve $c=1+\tau$ is shown dashed. There are three additional critical values 


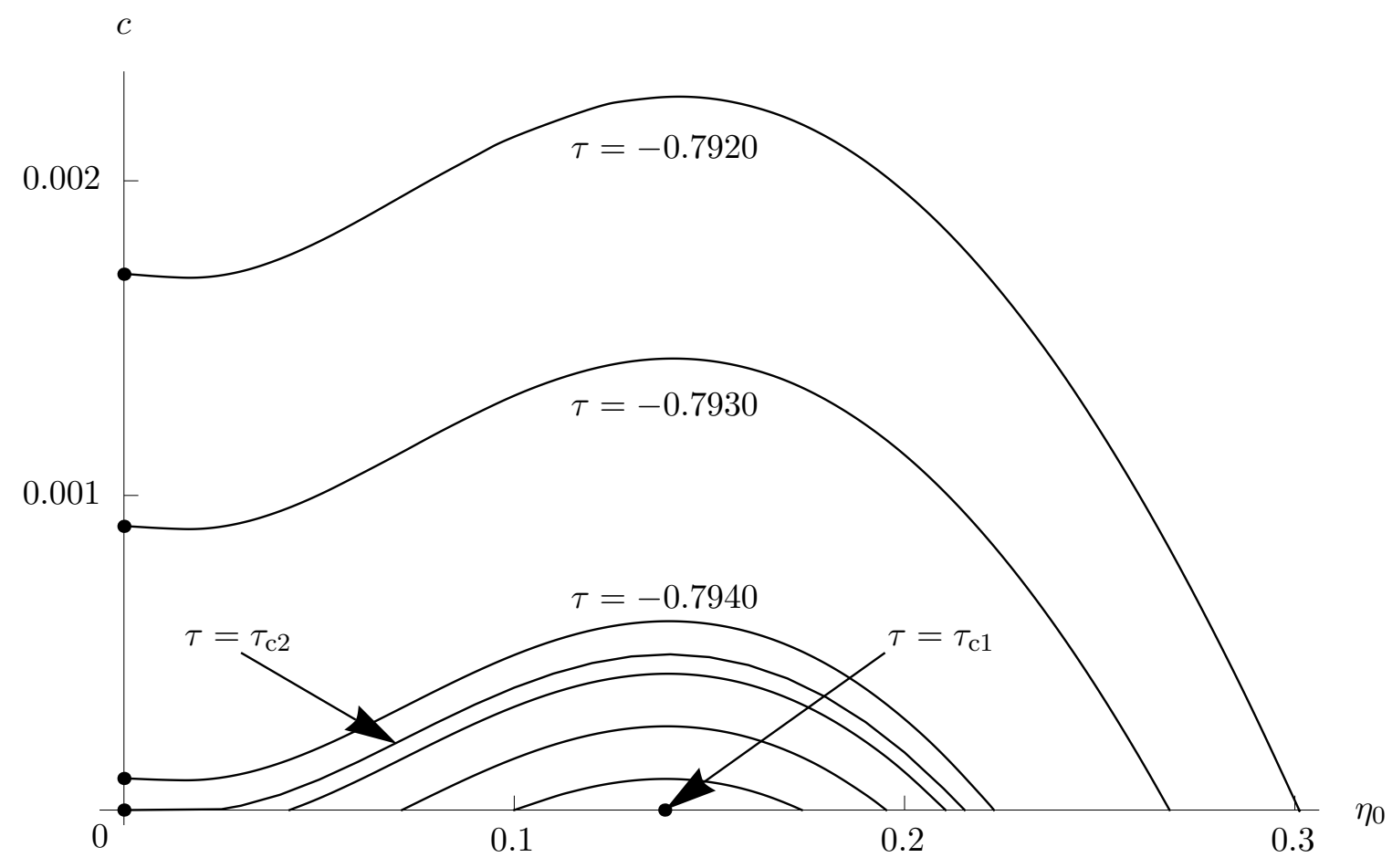

FIG. 5: Enlargement of Fig. 4, showing $c$ as a function of $\eta_{0}$ for $\tau=-0.7920,-0.7930,-0.7940, \tau_{\mathrm{c} 2} \simeq-0.7941$, $-0.7942,-0.7944,-0.7946$ and $\tau_{\mathrm{c} 1} \simeq-0.7947$.

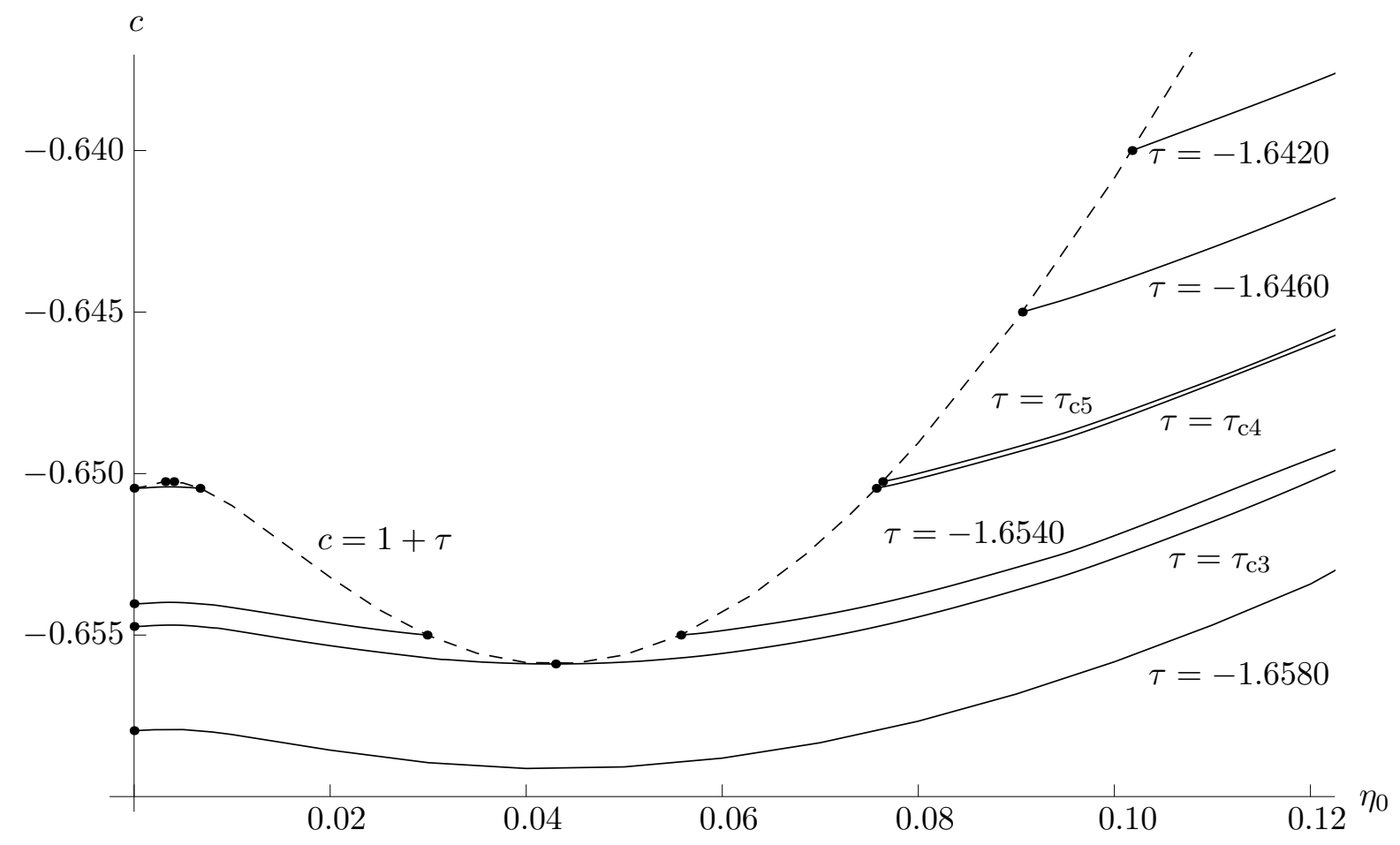

FIG. 6: Enlargement of Fig. 4, showing $c$ as a function of $\eta_{0}$ for various values of $\tau$, including $\tau=\tau_{\mathrm{c} 3} \simeq-1.6547$, $\tau=\tau_{\mathrm{c} 4} \simeq-1.6504$ and $\tau=\tau_{\mathrm{c} 5} \simeq-1.6502$. The bounding curve $c=1+\tau$ is shown with a dashed line. 


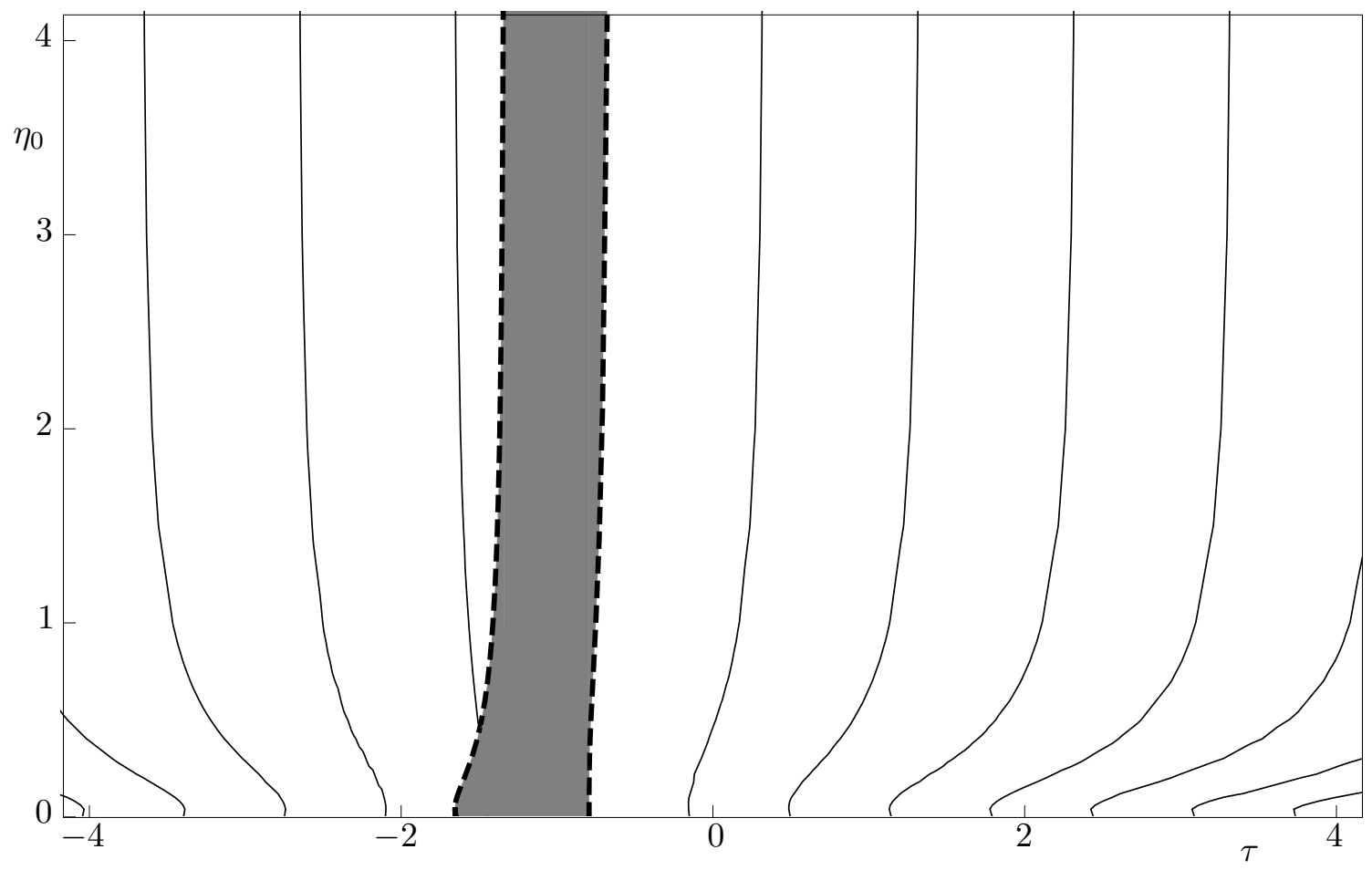

FIG. 7: Contour plot of $c$ in the $\tau-\eta_{0}$ plane; the contour values increase from $c=-5 / 2$ (leftmost) to $c=7 / 2$ (rightmost) in steps of $1 / 2$. There is no solution in the shaded region, given by $1+\tau<c<0$, the left boundary of which, $c=1+\tau$, is not a contour of $c$.

of $\tau$, namely $\tau=\tau_{\mathrm{c} 3} \simeq-1.6547$, corresponding to the solution curve that passes through the minimum on the bounding curve, $\tau=\tau_{\mathrm{c} 4} \simeq-1.6504$, corresponding to the (discontinuous) solution curve that intersects the bounding curve at $\eta_{0}=0$, and $\tau=\tau_{\mathrm{c} 5} \simeq-1.6502$, corresponding to the (discontinuous) solution curve that passes through the local maximum on the bounding curve. For $\tau \leq \tau_{\mathrm{c} 3}$ there is a solution $c$ for any value of $\eta_{0}$, whereas for $\tau_{\mathrm{c} 3}<\tau<-4 / 3$ there is a solution $c$ for sufficiently large $\eta_{0}$, as well as for an additional finite interval of $\eta_{0}$ values for $\tau_{\mathrm{c} 3}<\tau \leq \tau_{\mathrm{c} 5}$; for $\tau_{\mathrm{c} 4}<\tau<\tau_{\mathrm{c} 5}$ there is no solution for a sufficiently narrow dry patch.

In summary, Figs 3-6 show that there can be a solution $c$ (and hence the similarity solution (19) exists) if either $\tau \geq \tau_{\mathrm{c} 1}$ (in which case $c \geq 0$ ) or $\tau<-4 / 3$ (in which case $c<0$ ), but there is no solution for $-4 / 3 \leq \tau<\tau_{\mathrm{c} 1}$; for $\tau \geq-2 / 3$ and for $\tau \leq \tau_{\mathrm{c} 3}$ there is a solution for any value of $\eta_{0}$, but for other values of $\tau$ there is a solution only for restricted values of $\eta_{0}$.

Figure 7 shows a contour plot of $c$ in the $\tau-\eta_{0}$ parameter plane, obtained by interpolation of the data shown in Figs 3-6. In Fig. 7 the region $1+\tau<c<0$ in which there is no solution is 


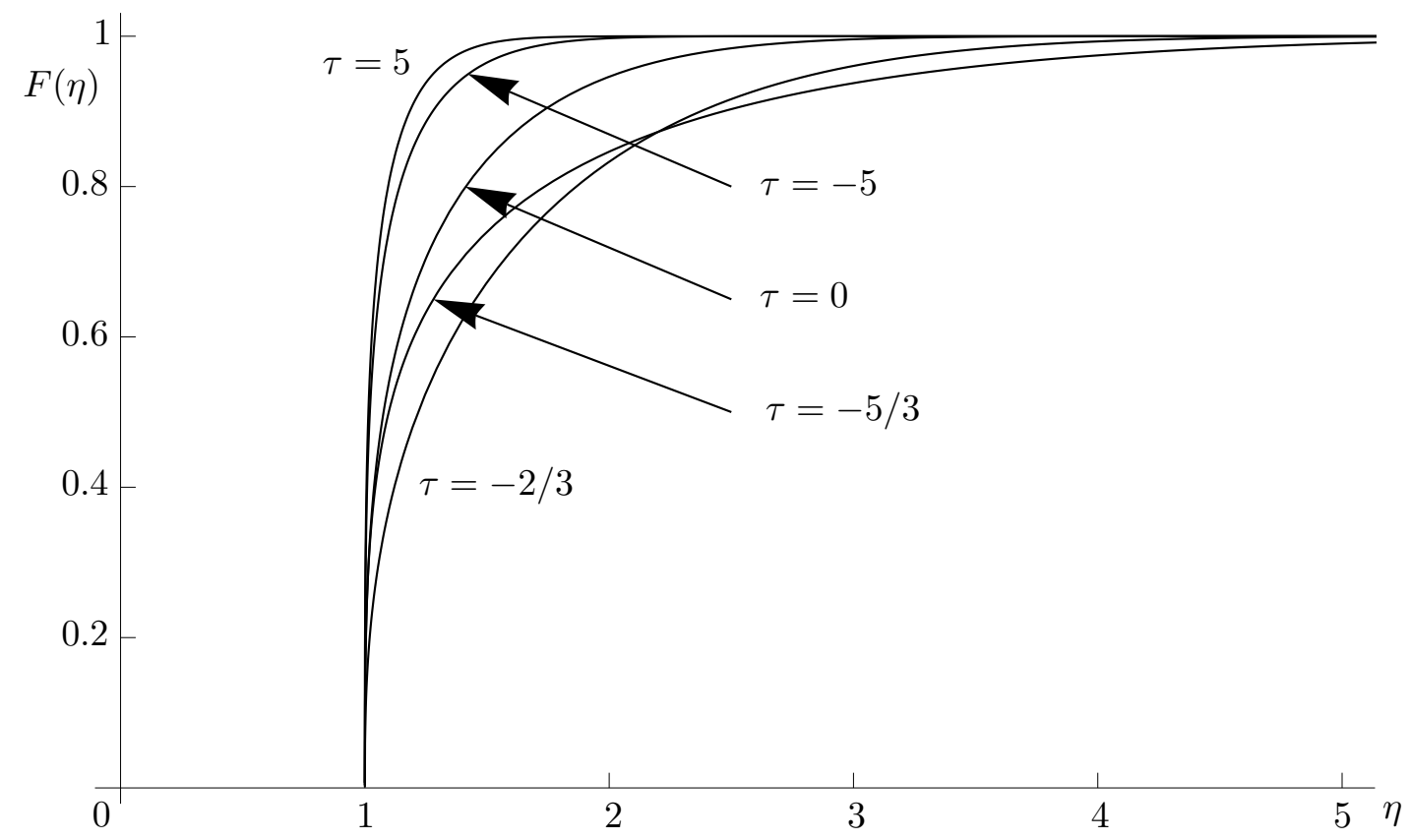

FIG. 8: Cross-sectional profiles $F(\eta)$ for $\tau=5,0,-2 / 3,-5 / 3$ and -5 , with $\eta_{0}=1$, for which $c \simeq 2.9611$, $0.4113,0.0494,-0.5560$ and -2.2820 , respectively.

shown shaded; note that the left boundary of this region, $c=1+\tau$, is not a contour of $c$.

Figure 8 shows examples of cross-sectional profiles $F(\eta)$ for $\tau=5,0,-2 / 3,-5 / 3$ and -5 in the case $\eta_{0}=1$, for which it was found that $c \simeq 2.9611,0.4113,0.0494,-0.5560$ and -2.2820 , respectively. In all the cases that we have examined numerically, $F$ increases monotonically with $\eta$, from $F=0$ at $\eta=\eta_{0}$ to $F \rightarrow 1$ as $\eta \rightarrow \infty$, consistent with the result in subsection III B. The curves for $\tau=10$ and $\tau=-10$ in Fig. 3 are nearly mirror images of each other in the $\eta_{0}$ axis (meaning that the speed of the dry patch up the substrate in the case $\tau=-10$ is almost the same as the speed of the dry patch down the substrate in the case $\tau=10$ ), reflecting the fact that for sufficiently large $|\tau|$ the gravity-driven ingredient in the flow is negligible in comparison with the shear-driven ingredient (associated with $\tau$ ), so that the behaviour in the limit $\tau \rightarrow \infty$ is the same as that in the limit $\tau \rightarrow-\infty$ except that the flow direction is reversed. For the same reason the cross-sectional profiles $F(\eta)$ for $\tau=5$ and $\tau=-5$ in Fig. 8 are somewhat similar to each other. The behaviour of the solutions in the purely surface-shear-stress-driven limit $|\tau| \rightarrow \infty$ will be discussed in more detail in $\S \mathrm{VI}$.

Figure 9 shows plots of (a) $q_{\text {area }}$ and (b) $q_{\text {flux }}$ (in terms of which the area difference $\triangle A$ and 


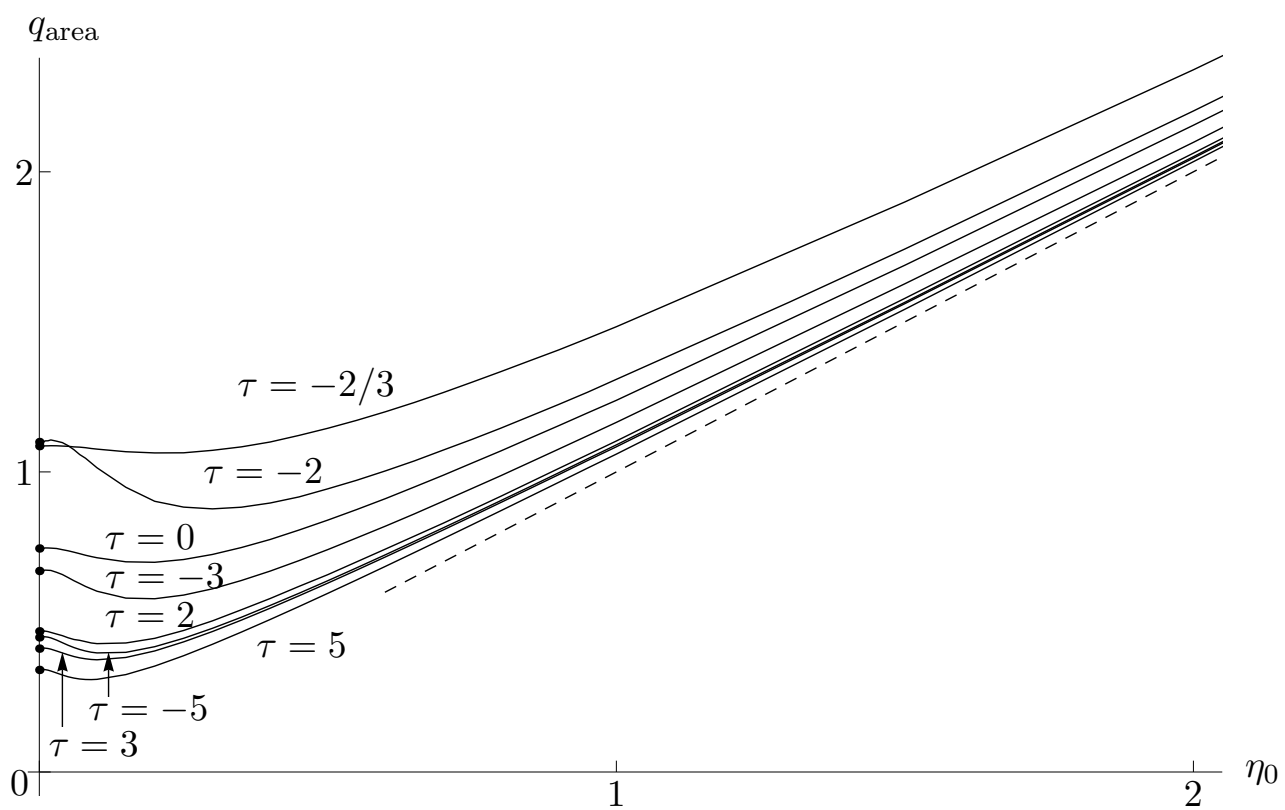

(a)

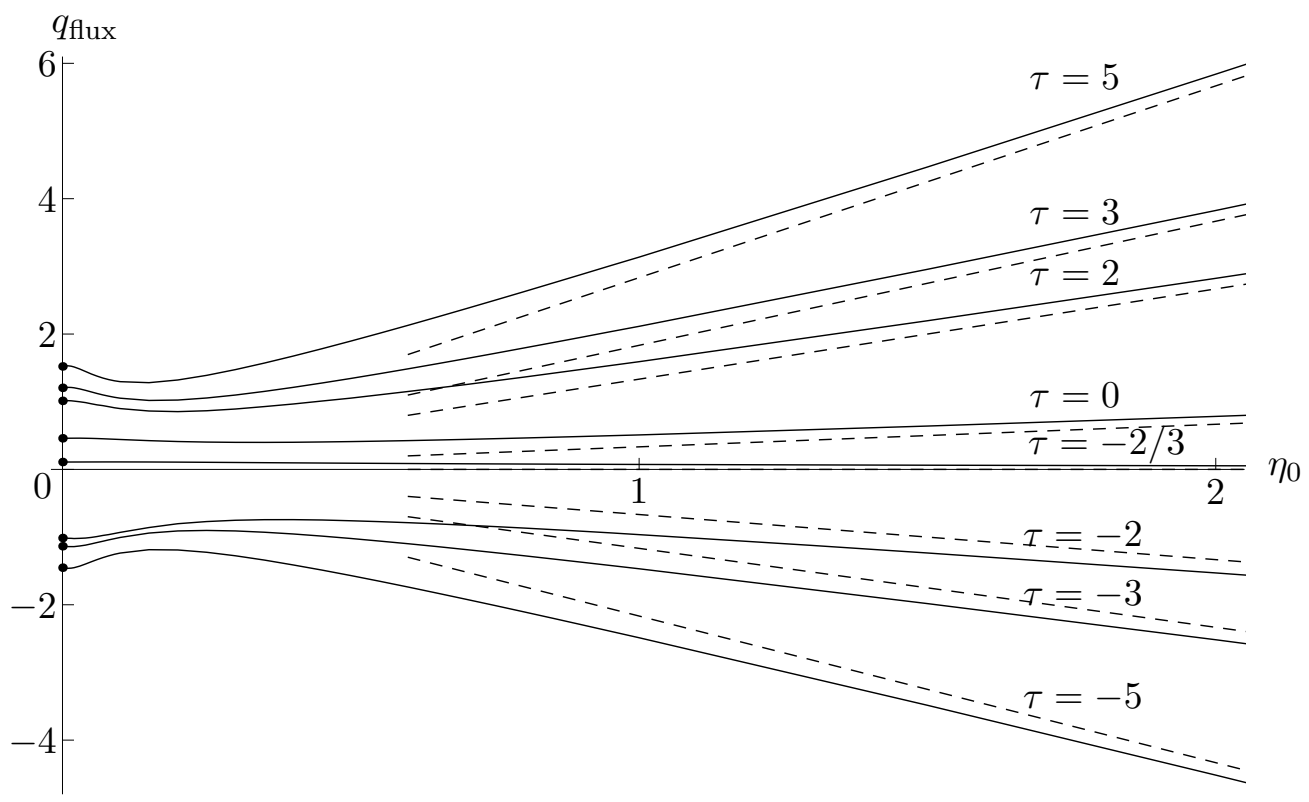

(b)

FIG. 9: Plots of (a) $q_{\text {area }}$ and (b) $q_{\text {flux }}$ as functions of $\eta_{0}$ for various values of $\tau$. The asymptotic forms in the limit $\eta_{0} \rightarrow \infty$ given later in (55), namely $q_{\text {area }} \sim \eta_{0}$ and $q_{\text {flux }} \sim(1 / 3+\tau / 2) \eta_{0}$, are shown with dashed lines.

the flux difference $\triangle Q$ are given by (37) and (39), respectively) as functions of $\eta_{0}$ for various values of $\tau$. In general, both $q_{\text {area }}$ and $q_{\text {flux }}$ are non-monotonic functions of $\eta_{0}$. (The dashed lines in Fig. 9 denote asymptotic results in the limit $\eta_{0} \rightarrow \infty$, described later in section IV D.) 


\section{B. Velocity and streamlines}

We now categorise the types of flow pattern that can occur in the transverse plane; the discussion here is analogous to that of Wilson and Duffy ${ }^{25}$ and Sullivan, Wilson, and Duffy ${ }^{26}$ for the rather different physical context of flow of a rivulet of fluid on an inclined substrate subject to a constant longitudinal surface shear stress.

If the prescribed surface shear stress acts down the substrate $(\tau \geq 0)$, in cooperation with the effect of gravity, then, as expected, the flow is downwards everywhere in the film (that is, $u \geq 0)$. If the prescribed surface shear stress acts up the substrate $(\tau<0)$, in opposition to the effect of gravity, then the competition between these opposing effects leads to several possibilities: if the upward surface shear stress is sufficiently weak (specifically, if $-1 / 2<\tau<0$ ) then the flow is downwards except in a finite region adjacent to the free surface near the contact line in which it is upwards (that is, $u<0$ ), if the upward surface shear stress is sufficiently strong (specifically, if $\tau \leq-1 / 2$ ) then the region of upflow extends to infinity, and if the upward surface shear stress is even stronger (specifically, if $\tau \leq-1$ ) then the flow is upwards everywhere. In all cases, sufficiently near to the contact line the flow is downwards if $\tau \geq 0$ and upwards if $\tau<0$. An analogous statement may be made concerning the local flux $\bar{u}$ given in (12): the (downward) contribution due to gravity varies like $h^{3}$ whereas the (upward or downward) contribution due to the surface shear stress varies like $h^{2}$, with the net effect that if $\tau \geq 0$ then the local flux is downwards everywhere $(\bar{u}>0)$, and if $\tau \leq-2 / 3$ then it is upwards everywhere $(\bar{u}<0)$, whereas in the intermediate case $-2 / 3<\tau<0$ it is upwards near the contact line but is downwards far from the contact line.

Figure 10 shows examples of each of the above forms of the flow in the transverse plane; in these plots, regions of upflow (downflow) are shown shaded (unshaded). Contours of the velocity $u$ are included in the plots. When $0<\tau<-1$ the contour on which $u=0$ (other than the substrate), given by $z=2(h+\tau)$, separates the shaded from the unshaded regions; this contour crosses the free surface where $h=-2 \tau$ and crosses the $\eta$ axis at the position where $h=-\tau$. Thus when $h<-\tau$ (and, in particular, near the contact line) the flow is 

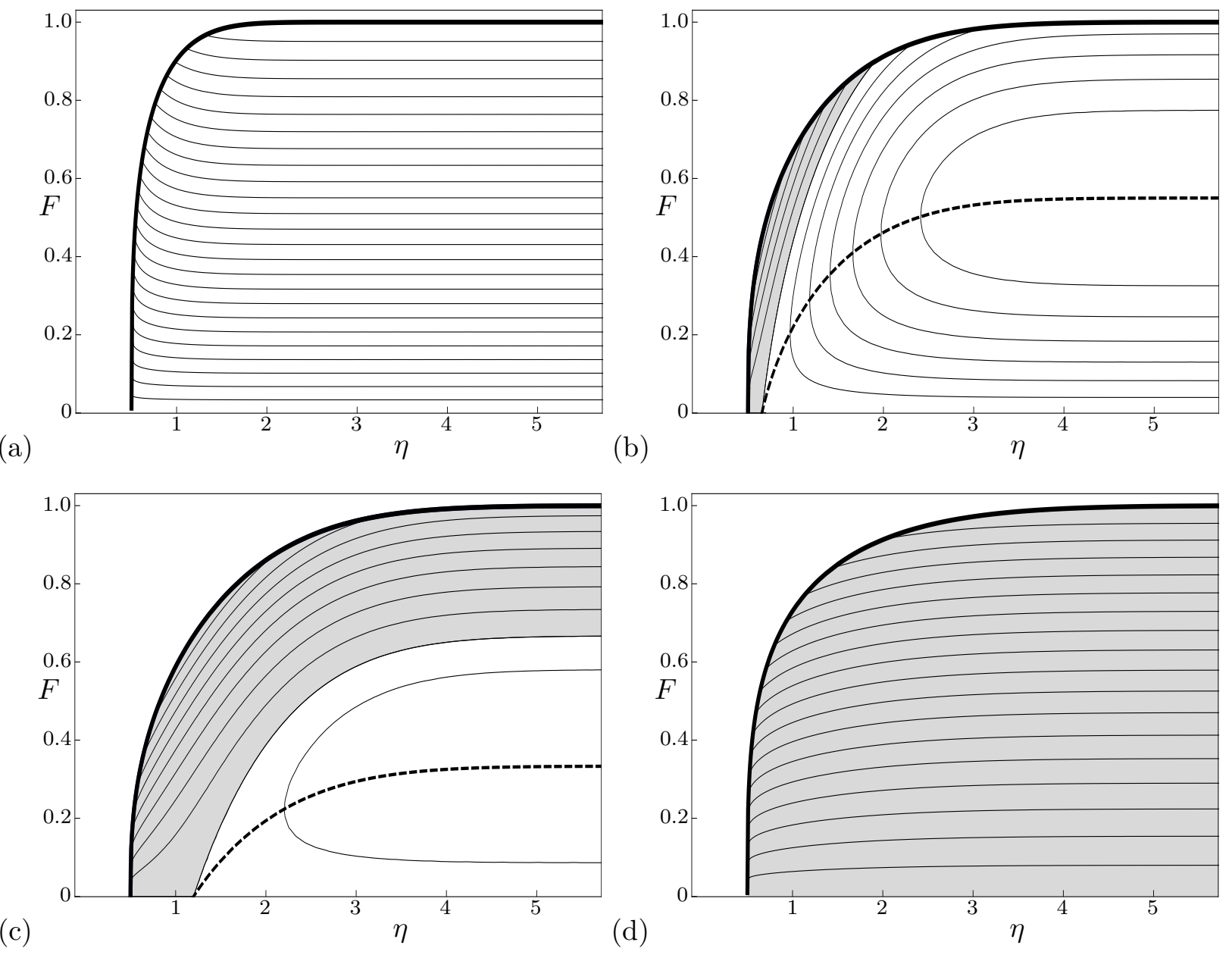

FIG. 10: Free surface profiles and contour plots of the $x$ velocity $u$ for (a) $\tau=2$, (b) $\tau=-0.45$, (c) $\tau=-2 / 3$ and (d) $\tau=-2$, with $\eta_{0}=1 / 2$ in each case. Regions of downflow $(u \geq 0)$ and of upflow $(u<0)$ are shown unshaded and shaded, respectively. The curves on which $\partial u / \partial z=0$, namely $z=h+\tau$, are shown with dashed lines.

always upwards, while if $h>-\tau$ (which requires $\tau>-1$ ) then the flow is downwards near the substrate, and if $h>-2 \tau$ (which requires $\tau>-1 / 2$ ) then the flow is downwards all the way across the film. Curves on which $u$ has a local maximum as a function of $z$ (so that $\partial u / \partial z=0)$, namely $z=h+\tau$, are shown with dashed lines in Fig. 10. If $\tau \geq 0$ then the maximum velocity $u_{\max }$ is given by $u_{\max }=(1 / 2)+\tau$, occurring on the free surface far from the dry patch, and the minimum velocity $u_{\min }$ is simply $u_{\min }=0$, occurring on the substrate. If $-1<\tau<0$ then $u_{\max }=(1+\tau)^{2} / 2$, occurring far from the dry patch, at height $z=1+\tau$ within the fluid, and $u_{\min }=-\tau^{2} / 2$, occurring on the free surface where $h=-\tau$. If $\tau \leq-1$ then $u_{\max }=0$, occurring on the substrate, and $u_{\min }=(1 / 2)+\tau$, occurring on the free surface far from the dry patch. 
Figure 11 shows examples of streamlines of the depth-integrated flow in a frame of reference moving with the dry patch, obtained from (35) in the case $\eta_{0}=1 / 2$ and $\tau=2$ (for which $c \simeq 1.6012$ ), plotted for (a) a sessile case and (b) a pendent case. The two examples shown in Fig. 11 (corresponding to flows of the types shown in parts (a) and (c) of Fig. 2, respectively) are mirror images of each other in the plane $X=0$, except that the direction of the depthintegrated flow in this moving reference frame is from bottom to top in both (a) and (b) (whereas in a fixed frame of reference the depth-integrated flow is downwards everywhere in both of these examples.)

\section{Asymptotic solution in the limit of a narrow dry patch, $\eta_{0} \rightarrow 0$}

It is enlightening to investigate the solutions in the asymptotic limits of a narrow dry patch, $\eta_{0} \rightarrow 0$, and a wide dry patch, $\eta_{0} \rightarrow \infty$, and, in particular, to compare them with numerical solutions; this we do in the present subsection and the next, respectively.

The limit $\eta_{0} \rightarrow 0$ corresponds physically to the case where the dry patch is narrow, so that to leading order there is a semi-infinite "dry line" on $\eta=0$ along which $h=0 .{ }^{27}$ In this limit we write

$$
\eta=\eta_{0}+\hat{\eta}, \quad F=\hat{F}(\hat{\eta}), \quad c=c_{0}
$$

then at leading order equation (21) reduces to

$$
\left(\hat{F}^{4}\right)^{\prime \prime}+6 S_{\infty} \hat{\eta}\left(\hat{F}^{2}+\tau \hat{F}-c_{0}\right) \hat{F}^{\prime}=0
$$

and the boundary conditions (23) and (24) give

$$
\hat{F}=0 \quad \text { at } \quad \hat{\eta}=0, \quad \hat{F}^{3} \hat{F}^{\prime} \rightarrow 0 \quad \text { as } \quad \hat{\eta} \rightarrow 0, \quad \hat{F} \rightarrow 1 \quad \text { as } \quad \hat{\eta} \rightarrow \infty
$$

At leading order in the limit $\hat{\eta} \rightarrow 0$ equations (44) and (45) have the asymptotic solution

$$
\hat{F} \sim\left(\frac{9 S_{\infty} c_{0} \hat{\eta}^{2}}{10}\right)^{\frac{1}{3}}
$$

for $S_{\infty} c_{0}>0$, but have no solution for $S_{\infty} c_{0}<0$. Equation (44) was solved for $\hat{F}$ numerically by means of a shooting method similar to that described in subsection IV A, subject to 
(a)

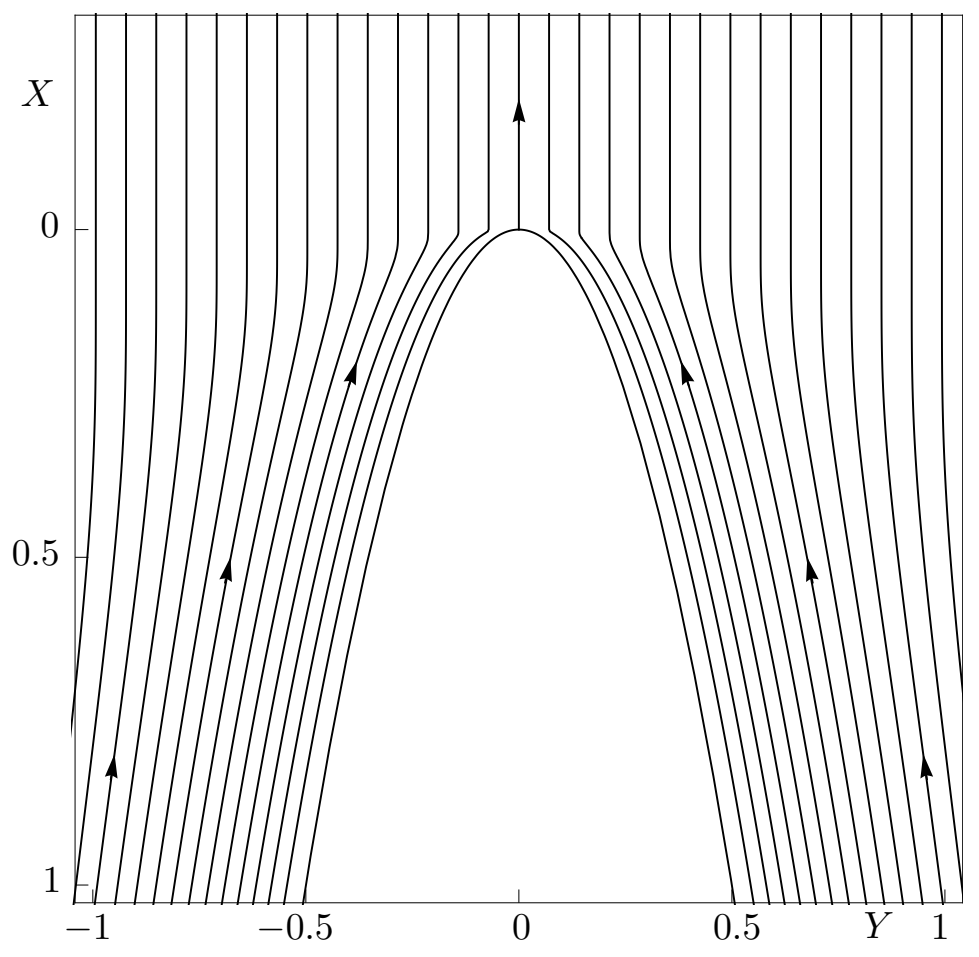

(b)

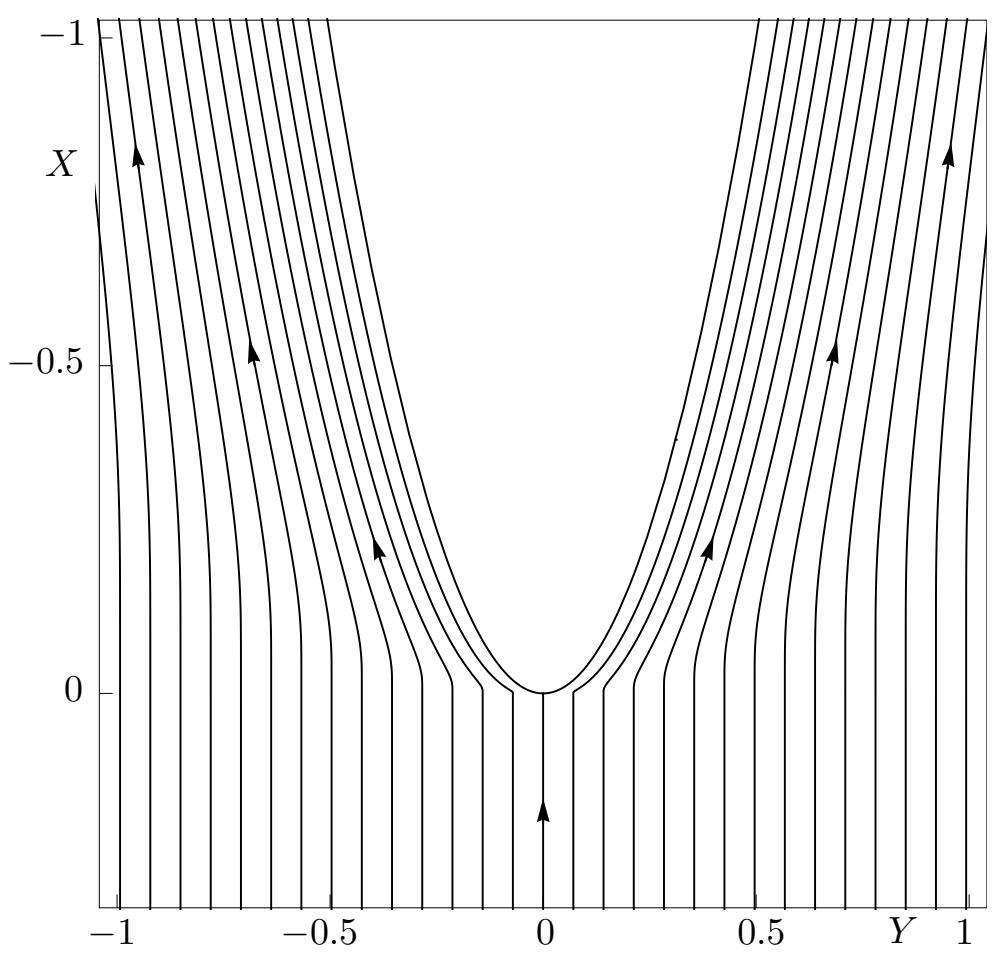

FIG. 11: Streamlines of the depth-integrated flow in a frame of reference moving with the dry patch, in the case $\eta_{0}=1 / 2$ and $\tau=2$ (for which $c \simeq 1.6012$ ), plotted for (a) a sessile case and (b) a pendent case. In both (a) and (b) the direction of flow in this moving reference frame is from bottom to top. 

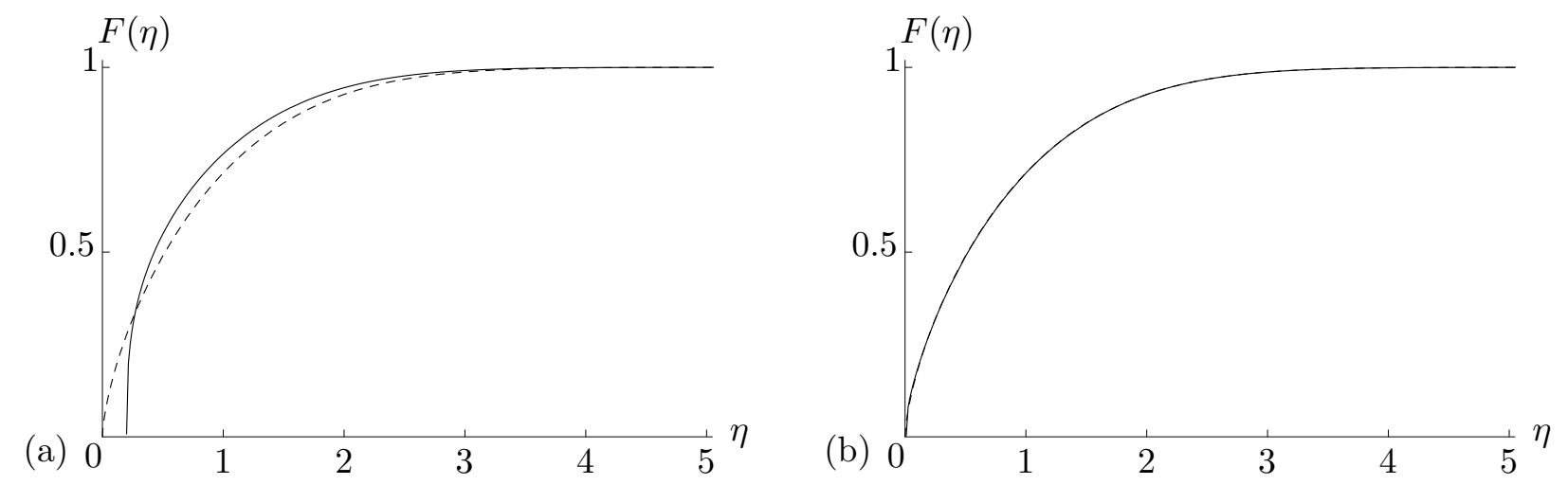

FIG. 12: Numerical solutions for $F$ of (21) in the cases (a) $\eta_{0}=0.2$ and (b) $\eta_{0}=10^{-2}$, with $\tau=0$ (shown with solid lines), together with the leading-order asymptotic solution $\hat{F}$ in the limit $\eta_{0} \rightarrow 0$ obtained by solving (44) subject to $(45)_{3}$ and (47) (shown with dashed lines). In part (b) the dashed line is virtually indistinguishable from the solid line.

approximated boundary conditions obtained from (46), namely

$$
\hat{F}(\delta)=\left(\frac{9 S_{\infty} c_{0} \delta^{2}}{10}\right)^{\frac{1}{3}}, \quad \hat{F}^{\prime}(\delta)=\frac{2}{3}\left(\frac{9 S_{\infty} c_{0}}{10 \delta}\right)^{\frac{1}{3}},
$$

where $0<\delta \ll 1$. Figure 12 shows a comparison between the numerical solution for $F$ of equation (21) and the leading-order asymptotic solution $\hat{F}$ in the cases $\eta_{0}=0.2$ and $\eta_{0}=10^{-2}$, with $\tau=0$ in each case (for which it was found that $c \simeq 0.5893$ and 0.61692 , respectively, and $\left.c_{0} \simeq 0.61689\right)$; the agreement in Fig. 12(b) is so good that the curves are virtually indistinguishable.

With this asymptotic solution the quantities $q_{\text {area }}$ and $q_{\text {flux }}\left(=c q_{\text {area }}\right)$ satisfy

$$
q_{\text {area }} \rightarrow \int_{0}^{\infty}(1-\hat{F}) \mathrm{d} \hat{\eta}, \quad q_{\text {flux }} \rightarrow c_{0} q_{\text {area }}
$$

at leading order in the limit $\eta_{0} \rightarrow 0$; these limiting values are indicated by dots in Fig. 9 .

\section{Asymptotic solution in the limit of a wide dry patch, $\eta_{0} \rightarrow \infty$}

The limit $\eta_{0} \rightarrow \infty$ corresponds physically to the case where the dry patch is wide. In this limit we write

$$
\eta=\eta_{0}+\frac{\tilde{\eta}}{\eta_{0}}, \quad F=\tilde{F}(\tilde{\eta}), \quad c=c_{\infty}
$$


then at leading order equation (21) reduces to

$$
\left(\tilde{F}^{4}\right)^{\prime \prime}+6 S_{\infty}\left(\tilde{F}^{2}+\tau \tilde{F}-c_{\infty}\right) \tilde{F}^{\prime}=0
$$

and the boundary conditions (23) and (24) give

$$
\tilde{F}=0 \quad \text { at } \quad \tilde{\eta}=0, \quad \tilde{F}^{3} \tilde{F}^{\prime} \rightarrow 0 \quad \text { as } \quad \tilde{\eta} \rightarrow 0, \quad \tilde{F} \rightarrow 1 \quad \text { as } \quad \tilde{\eta} \rightarrow \infty
$$

This is readily solved to give the implicit solution

$$
S_{\infty} \tilde{\eta}=\frac{(2+3 \tau)^{2}}{4+3 \tau} \log \frac{2+3 \tau+2 \tilde{F}}{2+3 \tau}-\frac{4}{4+3 \tau} \log (1-\tilde{F})-2 \tilde{F}, \quad c_{\infty}=U_{\infty}
$$

if $\left|\tau_{\infty}\right|>1 / 3$

$$
\tilde{\eta}=-2 \log (1-\tilde{F})-2 \tilde{F}, \quad c_{\infty}=0
$$

if $\tau_{\infty}=1 / 3$, and

$$
\tilde{\eta}=4 \log (1-\tilde{F})+\frac{2 \tilde{F}(2-\tilde{F})}{1-\tilde{F}}, \quad c_{\infty}=-\frac{1}{3}
$$

if $\tau_{\infty}=-1 / 3$, with $U_{\infty}$ as in (18). There is no solution in the limit $\eta_{0} \rightarrow \infty$ if $\left|\tau_{\infty}\right|<1 / 3$, consistent with the results shown in Figs 3-7. Since $c \rightarrow c_{\infty}=U_{\infty}$ in the limit $\eta_{0} \rightarrow \infty$, the curves in Figs 3 and 4 for $\left|\tau_{\infty}\right| \geq 1 / 3$ have the asymptotic forms $c \sim c_{\infty}=(1 / 3)+(\tau / 2)$ and the contours of $c$ in Fig. 7 approach the vertical asymptotes $\tau=2(c-1 / 3)$.

Figure 13 shows a comparison between the numerical solution for $F$ of equation (21) and the leading-order asymptotic solution $\tilde{F}$ in (52) in the cases $\eta_{0}=1$ and $\eta_{0}=5$, with $\tau=0$ in each case (for which it was found that $c \simeq 0.4113$ and 0.3395 , respectively, and for which $c_{\infty}=1 / 3$ ); the agreement in Fig. 13(b) is so good that the curves are virtually indistinguishable.

With this asymptotic solution we find that $q_{\text {area }}$ and $q_{\text {flux }}\left(=c q_{\text {area }}\right)$ satisfy

$$
q_{\text {area }} \sim \eta_{0}, \quad q_{\text {flux }} \sim U_{\infty} \eta_{0}
$$

at leading order in the limit $\eta_{0} \rightarrow \infty$; these asymptotic forms are shown with dashed lines in Fig. 9. 

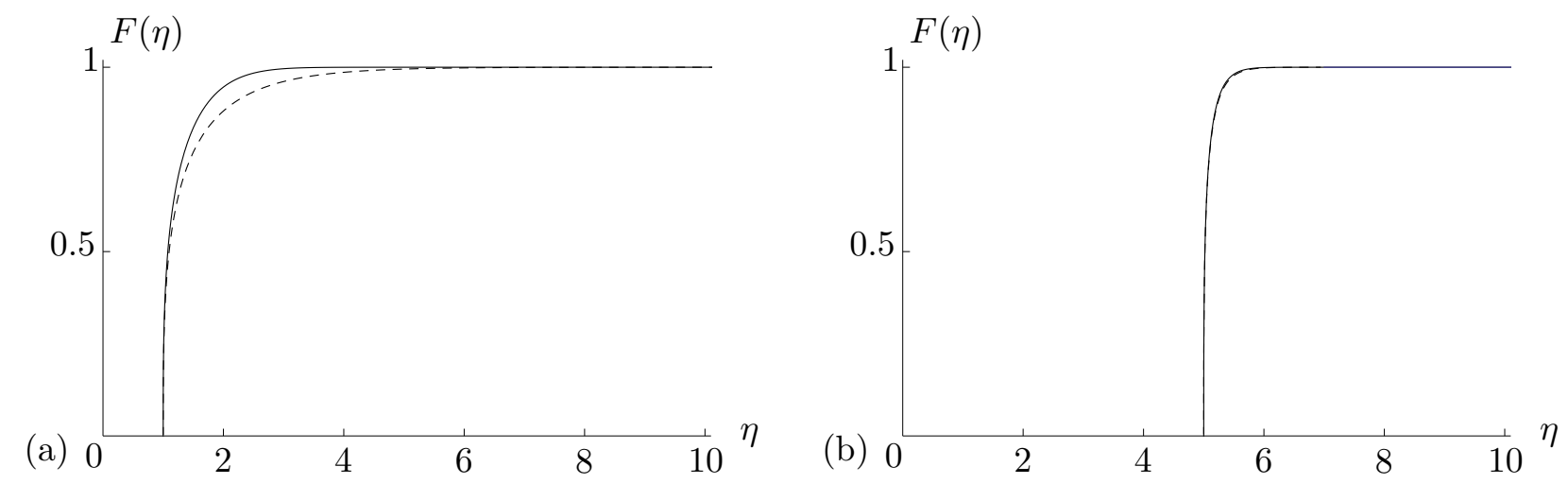

FIG. 13: Numerical solutions for $F$ of (21) in the cases (a) $\eta_{0}=1$ and (b) $\eta_{0}=5$, with $\tau=0$ (shown with solid lines), together with the leading-order asymptotic solution (52) in the limit $\eta_{0} \rightarrow \infty$ (shown with dashed lines). In part (b) the dashed line is virtually indistinguishable from the solid line.

\section{PURELY GRAVITY-DRIVEN FLOW}

Probably of most interest is the case of purely gravity-driven flow, with no surface shear stress (that is, $\tau=0$, so that $\tau_{\infty}=1$ and hence $S_{\infty}=+1$ ). In this case, as expected, the flow is downwards everywhere (that is, $u \geq 0$ ), and the dry patch always moves downwards (that is, $c>0$ ), and has the forms sketched in parts (a) and (c) of Fig. 2 in the sessile and pendent cases, respectively.

Figure 14 is the main result, showing a plot of the velocity of the dry patch, $c$, as a function of $\eta_{0}$ for this case; the inset in the figure shows details of the behaviour for small $\eta_{0}$. Figure 14 shows that $c$ is a single-valued function of $\eta_{0}$, but behaves non-monotonically; specifically, as is clear from the inset, $c$ decreases from its value $c=c_{0} \simeq 0.6169$ when $\eta=0$ to a (local) minimum value $c=c_{\min } \simeq 0.6167$ when $\eta_{0} \simeq 0.0050$, then increases to a (global) maximum value $c=c_{\max } \simeq 0.6225$ when $\eta_{0} \simeq 0.0630$, and thereafter decreases monotonically towards the asymptotic value $c_{\infty}=1 / 3$ as $\eta_{0} \rightarrow \infty$. Thus the velocity of the dry patch satisfies $1 / 3<c \leq c_{\max }$ for any value of $\eta_{0}$, a more restricted range than the general condition $0<c<1$ obtained from (29). Moreover, for a given value of $c$ there is one corresponding value of $\eta_{0}$ if either $c=c_{\max }$ or $1<c<c_{\min }$, two if either $c_{0}<c<c_{\max }$ or $c=c_{\min }$, three if $c_{\min }<c \leq c_{0}$, and none if either $c>c_{\max }$ or $c \leq 1$; thus there can be zero, one, two or three dry patches that 


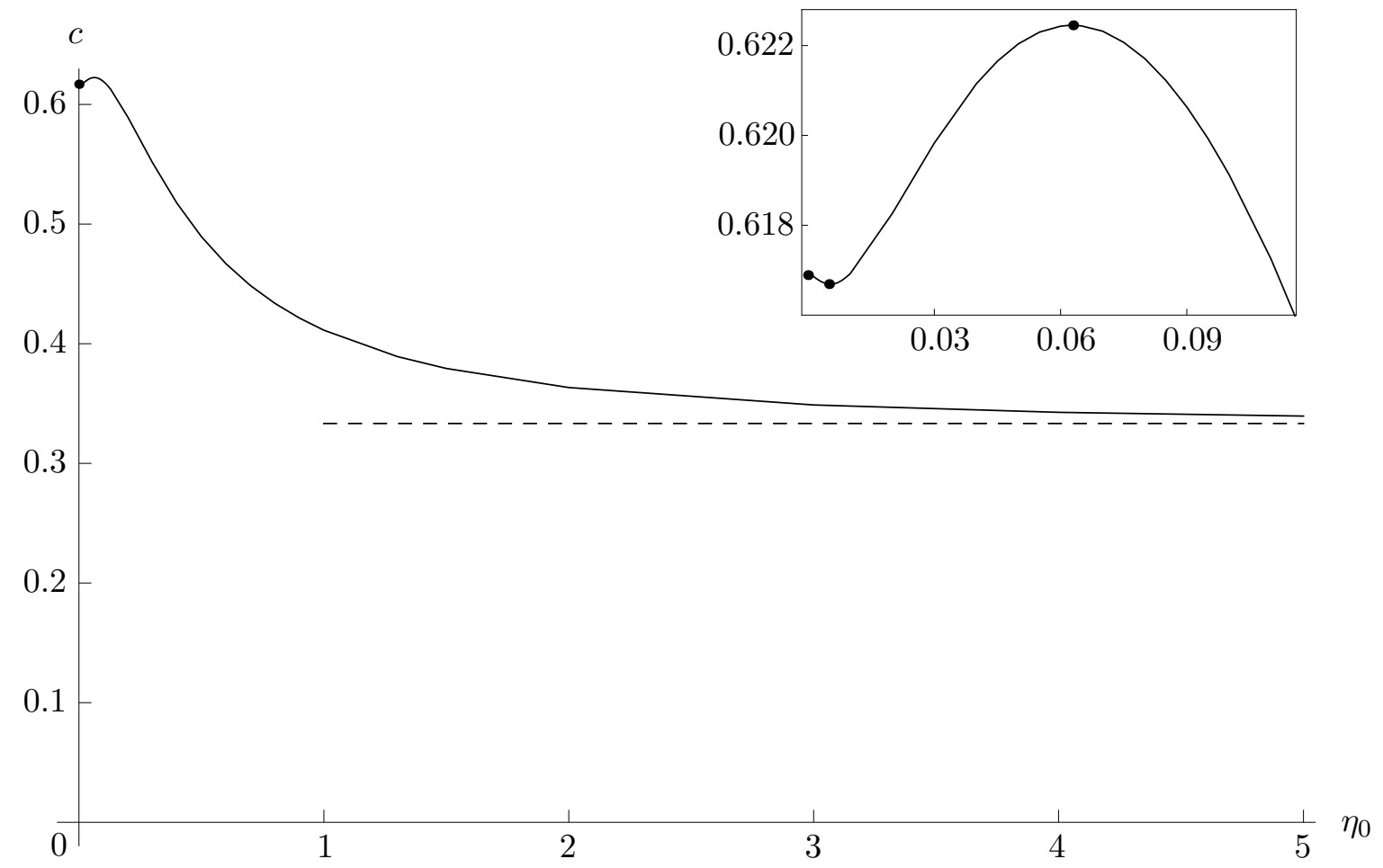

FIG. 14: Plot of $c$ as a function of $\eta_{0}$ for purely gravity-driven flow $(\tau=0)$, together with the asymptotic value $c=c_{\infty}=1 / 3$ in the limit $\eta_{0} \rightarrow \infty$ (shown with a dashed line). The inset shows an enlargement of the behaviour near $\eta_{0}=0$; the point $c=c_{0} \simeq 0.6169$ at $\eta_{0}=0$ is shown as a dot, as are the (local) minimum $c=c_{\min } \simeq 0.6167$ at $\eta_{0} \simeq 0.0050$ and the (global) maximum $c=c_{\max } \simeq 0.6225$ at $\eta_{0} \simeq 0.0630$.

move at a given velocity $c$.

Figure 15 shows examples of cross-sectional profiles $F(\eta)$ for the cases $\eta_{0}=0.1,1,3,5$ and 10 (for which it was found that $c \simeq 0.6191,0.4113,0.3488,0.3395$ and 0.3350 , respectively), and Fig. 16 shows three-dimensional plots of the free-surface profiles $h$ in a sessile case with $\eta_{0}=1$ at times $t=0,5$ and 10.

When $\eta_{0}=0$ we find from (48) that $q_{\text {area }} \simeq 0.7449$ and $q_{\text {flux }}=c_{0} q_{\text {area }} \simeq 0.4595$. In the limit $\eta_{0} \rightarrow \infty$ equations (52) and (55) with $U_{\infty}=1 / 3$ give, in terms of the rescaled variables defined in (49),

$$
\tilde{\eta}=\log \frac{1+\tilde{F}}{1-\tilde{F}}-2 \tilde{F}, \quad c_{\infty}=\frac{1}{3}, \quad q_{\text {area }} \sim 3 q_{\text {flux }} \sim \eta_{0} ;
$$

the asymptotic value $c=c_{\infty}=1 / 3$ is included in Fig. 14 as a dashed line.

Figure 17 shows plots of $q_{\text {area }}$ and $q_{\text {flux }}$ as functions of $\eta_{0}$ in this case, together with the above 


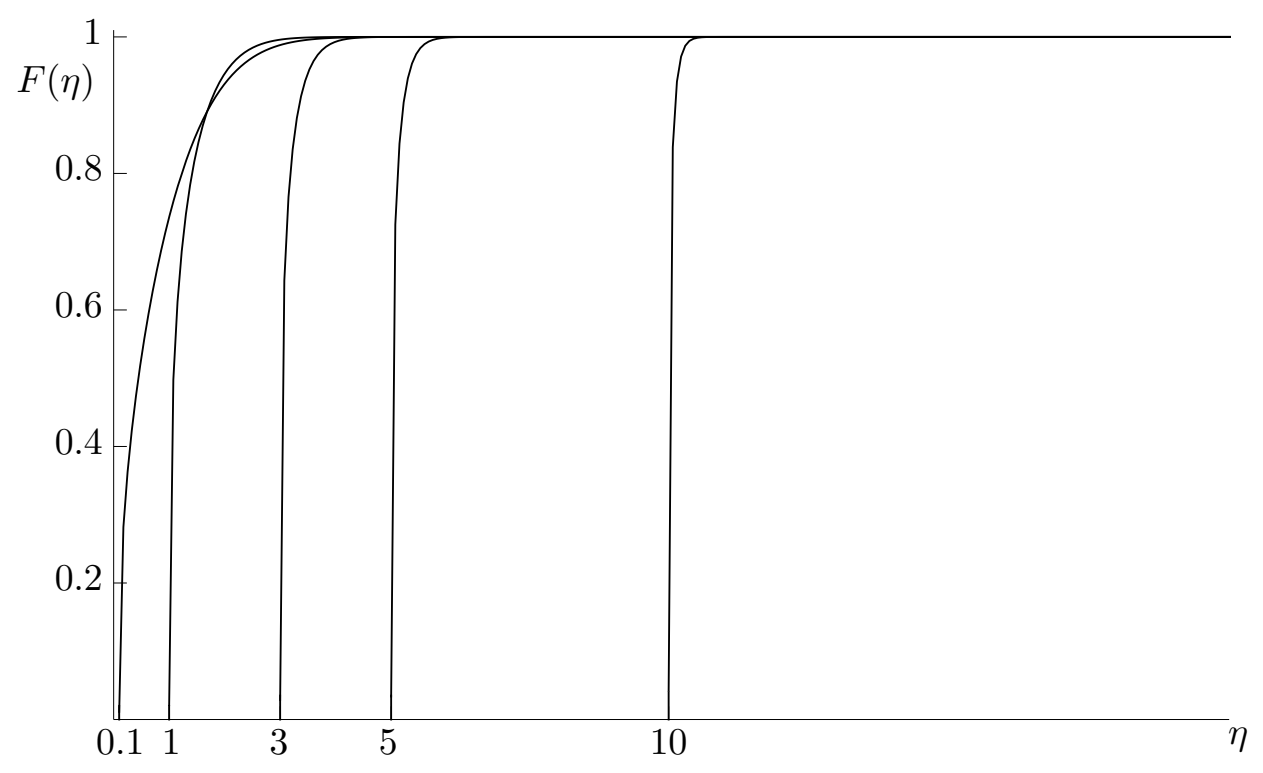

FIG. 15: Cross-sectional profiles $F(\eta)$ for purely gravity-driven flow $(\tau=0)$ for the cases $\eta_{0}=0.1,1,3,5$ and 10 , for which $c \simeq 0.6191,0.4113,0.3488,0.3395$ and 0.3350 , respectively.

values at $\eta_{0}=0$ (shown as dots) and the asymptotic forms (56) in the limit $\eta_{0} \rightarrow \infty$ (shown with dashed lines); both $q_{\text {area }}$ and $q_{\text {flux }}$ decrease from their values at $\eta_{0}=0$ to minimum values, and then increase monotonically to $\infty$ according to (56) as $\eta_{0} \rightarrow \infty$.

\section{PURELY SURFACE-SHEAR-STRESS-DRIVEN FLOW}

Purely surface-shear-stress-driven flow (that is, when the longitudinal component of gravity, $g \sin \alpha$, is negligible) is also of particular interest. This case corresponds to the limit $|\tau| \rightarrow \infty$ of the general case discussed earlier (in which $\tau$ was nondimensionalised with the shear-stress scale $\rho g \sin \alpha h_{\infty}$ ), but it is more natural and informative to treat it separately.

Since the longitudinal component of gravity, $g \sin \alpha$, is negligible, without loss of generality we now take $\tau$ to act down the substrate, so that $\tau>0$. Also the substrate may now be horizontal, and so we no longer exclude the inclination angles $\alpha=0$ and $\alpha=\pi$; however, we again exclude the case of a vertical substrate, $\alpha=\pi / 2$. We non-dimensionalise and scale variables as in (1), except that the factor $|\tan \alpha|$, wherever it appears, is now replaced by $\tau / \rho g h_{\infty}|\cos \alpha|$ (arising from the ratio of the longitudinal driving effect of $\tau$ to the transverse 

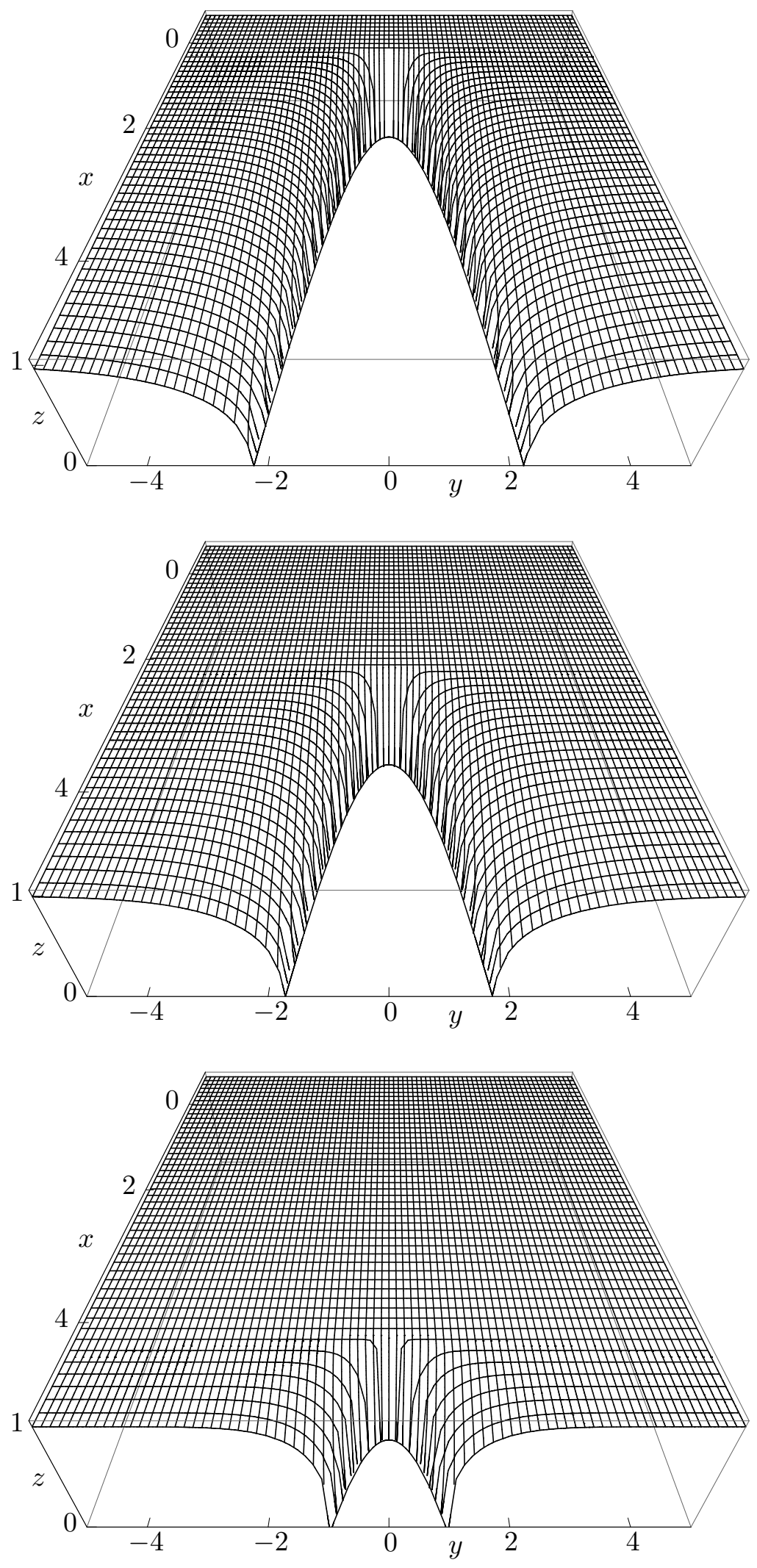

FIG. 16: Three-dimensional plots of the free-surface profiles $h$ for purely gravity-driven flow $(\tau=0)$ in a sessile case with $\eta_{0}=1$ (for which $c \simeq 0.4113$ ) at times $t=0,5$ and 10 . 


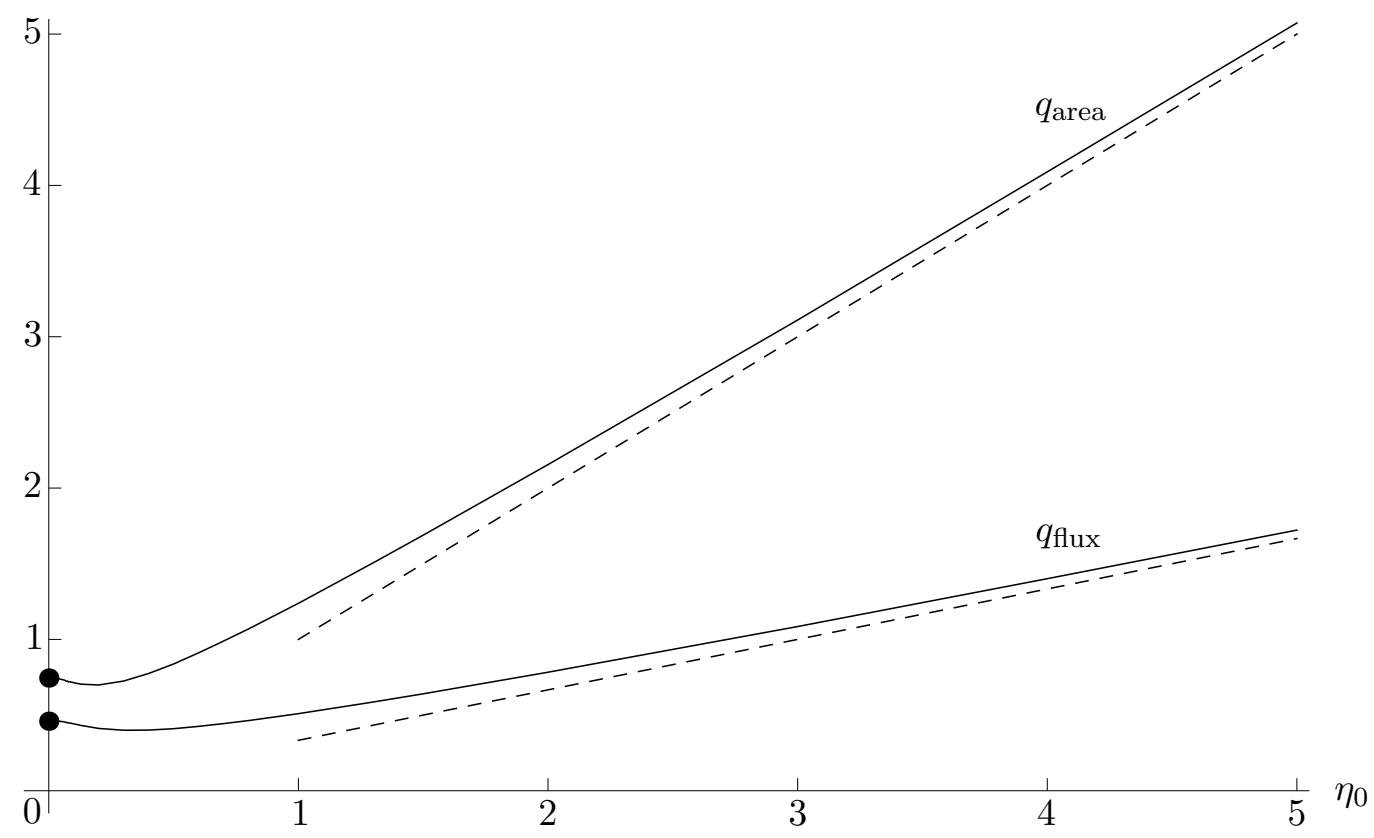

FIG. 17: Plot of $q_{\text {area }}$ (upper curve) and $q_{\text {flux }}\left(=c q_{\text {area }}\right)$ (lower curve) as functions of $\eta_{0}$ for purely gravitydriven flow $(\tau=0)$, together with the values $q_{\text {area }} \simeq 0.7449$ and $q_{\text {flux }} \simeq 0.4595$ at $\eta_{0}=0$ (shown as dots) and the asymptotic forms $q_{\text {area }} \sim \eta_{0}$ and $q_{\text {flux }} \sim \eta_{0} / 3$ in the limit $\eta_{0} \rightarrow \infty$ (shown with dashed lines).

spreading effect due to gravity), and now the appropriate velocity scale is the one associated with steady surface-shear-stress-driven flow of a film of uniform thickness $h_{\infty}$, namely $U_{\mathrm{S}}=\tau h_{\infty} / \mu$. In non-dimensional terms we now have $\tau=\tau_{\infty}=1$ and $S_{\infty}=+1$.

At leading order in $\epsilon$ the governing continuity and Navier-Stokes equations again give (2) and (3), except that $(3)_{1}$ is replaced by simply $u_{z z}=0$. The solution of these equations subject to (4)-(7) is as in (8) and (10) for $p$ and $v$, but (9) and (11) simplify to

$$
u=z, \quad w=\frac{S_{g}}{2}\left(h h_{y y}+h_{y}^{2}-\frac{h_{y y} z}{3}\right) z^{2}
$$

showing, in particular, that the flow is downwards everywhere (that is, $u \geq 0$ ), in the direction of the surface shear stress $\tau$, as expected. The local flux $\bar{u}$ is simply $\bar{u}=h^{2} / 2$, and $\bar{v}$ is again given by $\bar{v}=-S_{g} h^{3} h_{y} / 3$, and hence (13) is replaced by

$$
h_{t}=\frac{S_{g}}{3}\left(h^{3} h_{y}\right)_{y}-\frac{1}{2}\left(h^{2}\right)_{x} .
$$

Equation (58) has a travelling-wave similarity solution of the form (19) with $S_{\infty}=+1$, again representing a steadily translating dry patch. The resulting ordinary differential equation for 
$F(\eta)$, replacing $(21)$, is $^{28}$

$$
\left(F^{4}\right)^{\prime \prime}+6 \eta(F-c) F^{\prime}=0
$$

to be solved subject to the contact-line conditions (23) and the far-field condition (24).

Near the contact line $\eta=\eta_{0}$ the behaviour of $F$ is given by $(25)$ with $S_{\infty}=+1$ and $c>0$; the fact that $c>0$ shows that, as expected, the dry patch always moves downwards (that is, in the direction of $\tau$ ), and therefore has the forms sketched in parts (a) and (c) of Fig. 2 in the sessile and pendent cases, respectively. Also $F$ has the far-field behaviour (27) with $\tau_{\infty}=1, S_{\infty}=+1$ and $c<1$ in the limit $\eta \rightarrow \infty$, showing, in particular, that $c$ must satisfy the condition $0<c<1$. Note that since there is now no solution with $c \leq 0$, there are no results analogous to (26) and (28) in this case.

The effective stream function $\Psi$ in a frame of reference moving with the dry patch, defined in (34), takes the form

$$
\Psi= \begin{cases}\sqrt{S_{g} X}\left\{\frac{2 F^{3} F^{\prime}}{3}+\eta\left(\frac{F^{2}}{2}-c F\right)\right\} & \text { if } S_{g} X \geq 0 \\ \left(\frac{1}{2}-c\right) Y & \text { if } S_{g} X<0\end{cases}
$$

replacing (35). In addition, $\triangle A$ and $\triangle Q$ are again given by (37) and (40), with $q_{\text {area }}=I_{1}$ and $q_{\text {flux }}=c q_{\text {area }}=I_{2} / 2$.

\section{A. Solutions for $F(\eta)$ and $c$}

Equation (59) was solved numerically subject to (42) for the cross-sectional profile $F=F(\eta)$ and the velocity of the dry patch, $c$, by means of the same procedure as in Section IV A.

Figure 18 is the main result, showing a plot of $c$ as a function of $\eta_{0}$; again the inset in the figure shows details of the behaviour for small $\eta_{0}$. As Fig. 18 shows, the behaviour of $c$ in this case is qualitatively similar to that in the case of purely gravity-driven flow, shown in Fig. 14. It is found that $c$ decreases from its value $c=c_{0} \simeq 0.7712$ when $\eta_{0}=0$, to a (local) minimum value $c=c_{\min } \simeq 0.7711$ when $\eta_{0} \simeq 0.0050$, then increases to a (global) maximum value $c=c_{\max } \simeq 0.7751$ when $\eta_{0} \simeq 0.0550$, and thereafter decreases monotonically towards the 


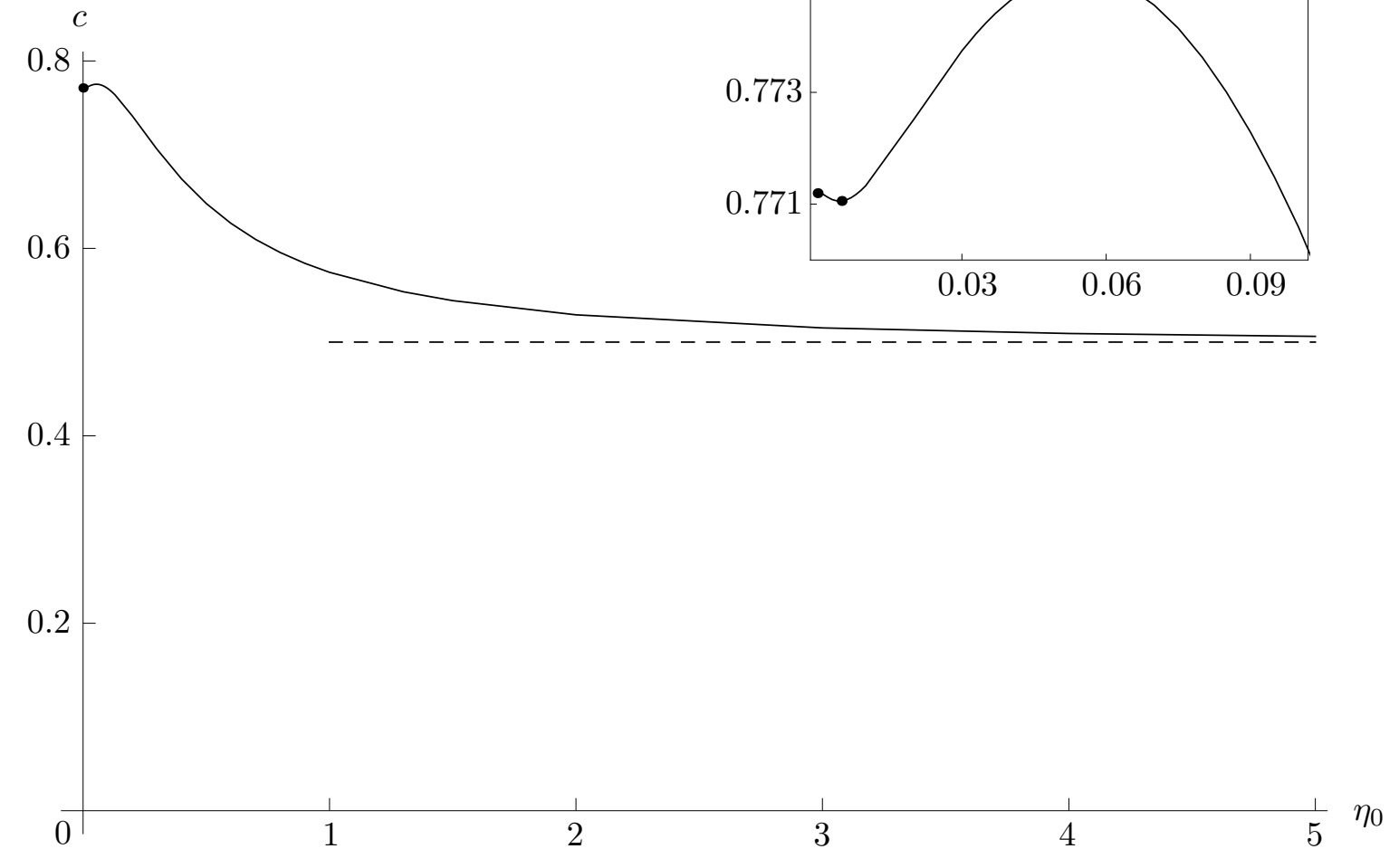

FIG. 18: Plot of $c$ as a function of $\eta_{0}$ for purely surface-shear-stress-driven flow, together with the asymptotic value $c=c_{\infty}=1 / 2$ in the limit $\eta_{0} \rightarrow \infty$ (shown with a dashed line). The inset shows an enlargement of the behaviour near $\eta_{0}=0$; the point $c=c_{0} \simeq 0.7712$ at $\eta_{0}=0$ is shown as a dot, as are the (local) minimum $c=c_{\min } \simeq 0.7711$ at $\eta_{0} \simeq 0.0050$ and the (global) maximum $c=c_{\max } \simeq 0.7751$ at $\eta_{0} \simeq 0.0550$.

asymptotic value $c_{\infty}=1 / 2$ as $\eta_{0} \rightarrow \infty$. Thus $c$ satisfies $1 / 2<c \leq c_{\max }$ for any value of $\eta_{0}$, again a more restricted range than the general condition $0<c<1$ obtained above; also there can again be zero, one, two or three dry patches that move at a given velocity $c$.

Figure 19 shows examples of cross-sectional profiles $F(\eta)$ for the cases $\eta_{0}=0.1,1,3,5$ and 10 (for which it was found that $c \simeq 0.7706,0.5745,0.5151,0.5061$ and 0.5016 , respectively). As the figure shows, the solutions for $F(\eta)$ in this case are qualitatively similar to those in the case of purely gravity-driven flow, shown in Fig. 15. Yatim ${ }^{29}$ gives three-dimensional plots of the free-surface profiles $h$ at different times; these plots are qualitatively similar to those in the case of purely gravity-driven flow, shown in Fig. 16, and so are omitted for brevity. 


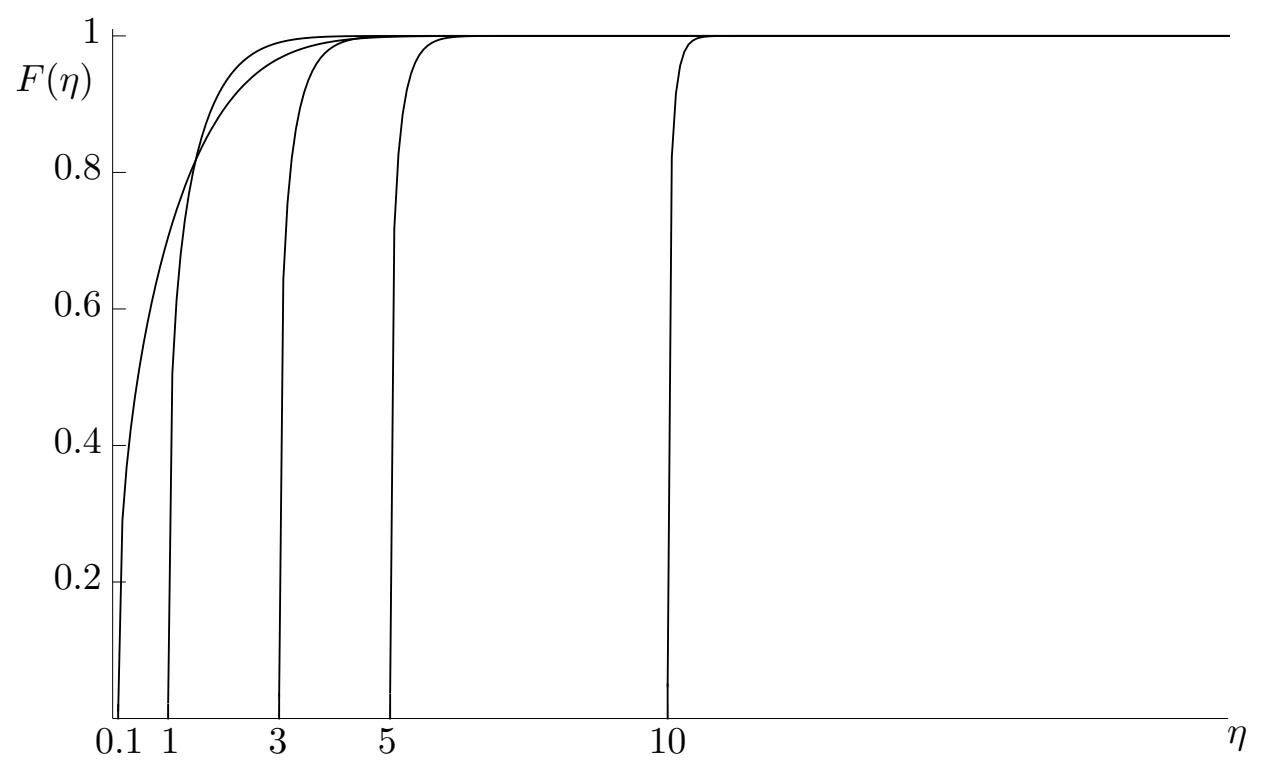

FIG. 19: Cross-sectional profiles $F(\eta)$ for purely surface-shear-stress-driven flow for the cases $\eta_{0}=0.1,1,3,5$ and 10 , for which $c \simeq 0.6191,0.4113,0.3488,0.3395$ and 0.3350 , respectively.

\section{B. Asymptotic solution in the limit of a narrow dry patch, $\eta_{0} \rightarrow 0$}

In the limit of a narrow dry patch, $\eta_{0} \rightarrow 0$, we again write $\eta, F$ and $c$ in the form (43); then at leading order equation (59) reduces to

$$
\left(\hat{F}^{4}\right)^{\prime \prime}+6 \hat{\eta}\left(\hat{F}-c_{0}\right) \hat{F}^{\prime}=0
$$

to be solved subject to (45). At leading order in the limit $\hat{\eta} \rightarrow 0$, the solution of (61) and (45) for $\hat{F}$ again has the asymptotic form (46) at leading order, and so (61) was solved for $\hat{F}$ numerically by means of the method described in subsection IV C, subject to $(45)_{3}$ and the approximated boundary conditions (47) obtained from (46). From this numerical solution it was found that $c_{0} \simeq 0.7712$, confirming the value obtained from the solution of (59) subject to (23) and (24). Furthermore $q_{\text {area }}$ again satisfies $(48)$ in the limit $\eta_{0} \rightarrow 0$, leading to $q_{\text {area }} \simeq 0.8565$ and $q_{\text {flux }}=c_{0} q_{\text {area }} \simeq 0.6605$ when $\eta_{0}=0$. Yatim ${ }^{29}$ gives plots comparing numerical solutions for $F(\eta)$ and the leading-order asymptotic solution $\hat{F}(\hat{\eta})$; these plots are again qualitatively similar to those in the case of purely gravity-driven flow, shown in Fig. 12, and so are again omitted for brevity. 


\section{Asymptotic solution in the limit of a wide dry patch, $\eta_{0} \rightarrow \infty$}

In the limit of a wide dry patch, $\eta_{0} \rightarrow \infty$, we again write $\eta, F$ and $c$ in the form (49); then at leading order equation (59) reduces to

$$
\left(\tilde{F}^{4}\right)^{\prime \prime}+6\left(\tilde{F}-c_{\infty}\right) \tilde{F}^{\prime}=0
$$

which is readily solved subject to the boundary conditions (51) to give the implicit solution

$$
\frac{3}{4} \tilde{\eta}=-\tilde{F}-\frac{\tilde{F}^{2}}{2}-\log (1-\tilde{F}), \quad c_{\infty}=\frac{1}{2} .
$$

Yatim $^{29}$ gives plots comparing numerical solutions for $F(\eta)$ and the leading-order asymptotic

solution $\tilde{F}(\tilde{\eta})$ in $(63)$; these plots are again qualitatively similar to those in the case of purely gravity-driven flow, shown in Fig. 13, and so are again omitted for brevity.

\section{Cross-sectional area and volume flux}

Yatim $^{29}$ gives plots of $q_{\text {area }}$ and $q_{\text {flux }}$ as functions of $\eta_{0}$ in this case, together with the values $q_{\text {area }} \simeq 0.8565$ and $q_{\text {flux }}=\simeq 0.6605$ at $\eta_{0}=0$ and the asymptotic forms (55) with $U_{\infty}=1 / 2$ in the limit $\eta_{0} \rightarrow \infty$; these plots are again qualitatively similar to those in the case of purely gravity-driven flow, shown in Fig. 17, and so are again omitted for brevity.

\section{CONCLUSIONS}

We have obtained a novel family of three-dimensional travelling-wave similarity solutions of the form (19) describing a steadily translating slender dry patch in an infinitely wide thin fluid film on an inclined planar substrate, the flow being driven by gravity and/or a prescribed constant shear stress on its free surface. For both driving mechanisms, the dry patch has a parabolic shape, which may be concave up or concave down the substrate, as indicated in Fig. 2, and the film thickness increases monotonically away from the contact lines to its uniform far-field value. The two most practically important cases of purely gravity-driven flow and of purely surface-shear-stress-driven flow were analysed in detail. 
The family of solutions (19) is parameterised by $\eta_{0}$, a scaled measure of the width of the dry patch, which is not determined as part of the solution; determining the value of $\eta_{0}$ (perhaps via stability considerations, or via a criterion based on the behaviour of the dry patch near its apex) remains an open question.

If the surface shear stress $\tau$ either acts downwards $(\tau \geq 0)$ or acts upwards but is sufficiently weak $\left(\tau_{\mathrm{c} 1} \leq \tau<0\right.$, where $\left.\tau_{\mathrm{c} 1} \simeq-0.7947\right)$ then the dry patch moves down the substrate $(c \geq 0)$, whereas if the surface shear stress acts upwards and is sufficiently strong $(\tau<-4 / 3)$ then the dry patch moves up the substrate $(c<0)$. It is possible for the dry patch to be stationary $(c=0)$ only if the surface shear stress acts upwards sufficiently strongly that it counters the effect of gravity but not so strongly that it forces the dry patch to move upwards; specifically, the dry patch can be stationary only if $\tau$ satisfies $\tau_{\mathrm{c} 1} \leq \tau<-2 / 3$, and then for only particular values of $\eta_{0}$. In particular, the dry patch cannot be stationary in purely gravity-driven flow $(\tau=0)$ nor in purely surface-shear-driven flow $(|\tau| \rightarrow \infty)$. Also for any prescribed value of $\eta_{0}$ the dry patch can be stationary for only one particular value of $\tau$ (which depends on $\eta_{0}$ ).

Rather unexpectedly, it was found that there is no solution of the type sought if the surface shear stress acts upwards and satisfies $-4 / 3 \leq \tau<\tau_{\mathrm{c} 1} \simeq-0.7947$; correspondingly there is no solution in the region of the $\eta_{0}-c$ parameter plane in Fig. 4 between the $\eta_{0}$ axis and the dashed curve. The physical explanation for this remains an open question.

The present solutions are valid for any value of the uniform film thickness far from the dry patch, $h_{\infty}(>0)$, showing that for these solutions there is, in general, no critical thickness or critical flux below which the dry patch is stationary but above which it is "swept away" by the flow. Notwithstanding this, the present solutions have physical features in common with dry patches studied experimentally (see, for example, Podgorski et al. ${ }^{10,11}$ ), but precise comparison is difficult because of the simplifying assumptions (particularly the neglect of surface tension) made here. ${ }^{30}$

Throughout the present analysis we have taken the substrate $z=0$ to be stationary. However, exactly the same kind of solution for a steadily translating dry patch on a substrate moving parallel to itself at constant velocity $U_{0} \mathbf{i}$ upwards or downwards (that is, with $U_{0}<0$ 
or $U_{0}>0$ ) may be obtained simply by means of a shift in the value of $c$ by $U_{0}$, that is, with $c$ replaced by $c-U_{0}$ in the above discussion. In particular, a solution (19) representing an unsteady flow with a dry patch translating steadily with velocity ci on a stationary substrate also provides a solution for a steady flow (with a stationary dry patch) on a substrate moving with velocity $-c$ i provided simply that $x-c t$ is replaced by $x$ in $\eta$.

Lastly, it is worth commenting on the question of travelling-wave similarity solutions of (13) of the form (19) for the rather different physical context of flow of a steadily translating symmetric slender rivulet occupying $|y| \leq a(x, t)$ on an inclined planar substrate, driven by gravity, a constant shear stress on its free surface and/or steady motion of the substrate. At first sight, such rivulet solutions seem feasible; however, the only solution of (21) satisfying $F(0)=F_{0}$ and $F^{\prime}(0)=0$ (where $F_{0}$ is a prescribed positive constant, representing the thickness of the rivulet at its middle) is $F(\eta) \equiv F_{0}$, which cannot satisfy the contact-line conditions $(23)$, showing that there are, in fact, no such rivulet solutions.

\section{ACKNOWLEDGMENTS}

The first author (YMY) wishes to thank the Ministry of Higher Education, Malaysia and Universiti Sains Malaysia for financial support via an Academic Staff Training Fellowship. Part of this work was undertaken while the third author (SKW) was a Visiting Fellow in the Department of Mechanical and Aerospace Engineering in the School of Engineering and Applied Science at Princeton University, USA, and part of it was undertaken while he was a Visiting

Fellow in the Oxford Centre for Collaborative Applied Mathematics (OCCAM), University of Oxford, Mathematical Institute, 24-29 St. Giles', Oxford OX1 3LB. This publication was based on work supported in part by Award No KUK-C1-013-04, made by King Abdullah University of Science and Technology (KAUST). 
APPENDIX: DERIVATION OF EQUATION (30) AND OF INEQUALITIES SATISFIED BY THE VELOCITY OF THE DRY PATCH, $c$

In this Appendix we obtain a first integral of (21), from which we derive (30), as well as certain inequalities relating $\tau, U_{\infty}$ and $c$ that were useful in determining the structure of the solutions described in subsection IV A.

Writing the differential equation (21) in the form

$$
\left(F^{4}\right)^{\prime \prime}+6 S_{\infty} \eta\left[(1-F)^{2}-(\tau+2)(1-F)+\tau+1-c\right] F^{\prime}=0
$$

integrating with respect to $\eta$ from $\eta_{0}$ to infinity, and using (23) and (27) we obtain

$$
\frac{1}{3} I_{3}+\frac{\tau}{2} I_{2}-c I_{1}=0
$$

where the constants $I_{n}(n=1,2,3)$ are as defined in (31). Thus the velocity of the dry patch, $c$, may be expressed in terms of integrals of $F$ as

$$
c=\frac{2 I_{3}+3 \tau I_{2}}{6 I_{1}}
$$

which is equation (30).

Since $0 \leq F<1$, from the definition (31) we have $0<I_{1}<I_{2}<I_{3}$, and so we deduce from (A.3) that if $\tau \geq 0$ then $c$ will always be positive, and that $c$ can be negative only if $\tau<0$; both of these statements are consistent with (29).

Equation (A.2) may be recast in the form

$$
\eta_{0}\left(U_{\infty}-c\right)+\int_{\eta_{0}}^{\infty}(1-F)\left(\frac{F^{2}}{3}+U_{\infty} F+U_{\infty}-c\right) \mathrm{d} \eta=0
$$

(with $U_{\infty}$ as in (18)), from which we deduce that if $U_{\infty} \geq 0$ then $c$ will satisfy $c>U_{\infty} \geq 0$, and that $c$ may satisfy $c \leq U_{\infty}$ only if $U_{\infty}<0$.

We also have

$$
2 I_{1}-I_{2}=\eta_{0}+\int_{\eta_{0}}^{\infty}(1-F)^{2} \mathrm{~d} \eta>0, \quad 3 I_{2}-2 I_{3}=\eta_{0}+\int_{\eta_{0}}^{\infty}(1-F)^{2}(2 F+1) \mathrm{d} \eta>0
$$


showing that $I_{2}<2 I_{1}$ and $I_{3}<3 I_{2} / 2$; therefore from (A.2) we deduce that if $\tau<-2$ then $c<1+\tau / 2<0$.

All the numerical results reported in sections IV-VI were found to be consistent with the above restrictions and with the inequalities in (29).

1 G. I. Taylor and D. H. Michael, "On making holes in a sheet of fluid," J. Fluid Mech. 58, 625-639 (1973).

2 J. A. Moriarty and L. W. Schwartz, "Dynamic considerations in the closing and opening of holes in thin liquid films," J. Colloid Interface Sci. 161, 335-342 (1993).

3 S. K. Wilson and E. L. Terrill, "The dynamics of planar and axisymmetric holes in thin fluid layers," in The Mechanics of Thin Film Coatings, Proceedings of the First European Coating Symposium, held in Leeds, 19th-22nd September 1995, World Scientific, Singapore (1996), pp. 288-297.

4 P. G. López, M. J. Miksis, and S. G. Bankoff, "Stability and evolution of a dry spot," Phys. Fluids 13, 1601-1614 (2001).

5 S. G. Bankoff, M. F. G. Johnson, M. J. Miksis, R. A. Schluter, and P. G. López, "Dynamics of a dry spot," J. Fluid Mech. 486, 239-259 (2003).

${ }^{6}$ D. E. Hartley and W. Murgatroyd, "Criteria for the break-up of thin liquid layers flowing isothermally over solid surfaces," Int. J. Heat Mass Transfer 7, 1003-1015 (1964).

7 W. Murgatroyd, "The role of shear and form forces in the stability of a dry patch in two-phase film flow," Int. J. Heat Mass Transfer 8, 297-301 (1965).

8 A. B. Ponter, G. A. Davies, T. K. Ross, and P. G. Thornley, "The influence of mass transfer on liquid film breakdown," Int. J. Heat Mass Transfer 10, 349-359 (1967).

9 S. D. R. Wilson, "The stability of a dry patch on a wetted wall," Int. J. Heat Mass Transfer 17, 1607-1615 (1974).

10 T. Podgorski, J.-M. Flesselles, and L. Limat, "Dry arches within flowing films," Phys. Fluids 11, 845-852 (1999).

11 T. Podgorski, J.-M. Flesselles, and L. Limat, "Courbure de la frontière d'une zone sèche dans un film en écoulement (Curvature of a dry patch boundary in a flowing film)," C. R. Acad. Sci. Paris 
Sér. IV Physics 2, 1361-1367 (2001).

12 E. Rio, A. Daerr, and L. Limat, "Probing with a laser sheet the contact angle distribution along a contact line," J. Colloid Interface Sci. 269, 164-170 (2004).

13 E. Rio and L. Limat, "Wetting hysteresis of a dry patch left inside a flowing film," Phys. Fluids 18, $032102(2006)$.

14 J. Sébilleau, L. Lebon, and L. Limat, "Stability of a dry patch in a viscous flowing film," Eur. Phys. J. Special Topics 166, 139-142 (2009).

15 S. K. Wilson, B. R. Duffy, and S. H. Davis, "On a slender dry patch in a liquid film draining under gravity down an inclined plane," Euro. J. Appl. Math. 12, 233-252 (2001).

16 R. P. Agarwal and D. O'Regan, "Infinite interval problems arising in the model of a slender dry patch in a liquid film draining under gravity down an inclined plane," Methods Appl. Anal. 10, 363-376 (2003).

17 D. Holland, S. K. Wilson, and B. R. Duffy, "Similarity solutions for slender dry patches with thermocapillarity," J. Eng. Math. 44, 369-394 (2002).

18 Y. M. Yatim, B. R. Duffy, and S. K. Wilson, "Similarity solutions for unsteady shear-stress-driven flow of a power-law fluid: slender rivulets and dry patches," J. Eng. Math. 73, 53-69 (2012).

19 Y. M. Yatim, B. R. Duffy, S. K. Wilson, and R. Hunt, "Similarity solutions for unsteady gravitydriven slender rivulets," Q. J. Mech. Appl. Math. 64, 455-480 (2011).

20 Y. M. Yatim, S. K. Wilson, and B. R. Duffy, "Unsteady gravity-driven slender rivulets of a powerlaw fluid," J. Non-Newtonian Fluid Mech. 165, 1423-1430 (2010).

21 S. I. Betelú and J. A. Diez, "A two-dimensional similarity solution for capillary driven flows," Physica D 126, 136-140 (1999).

22 D. Bonn, J. Eggers, J. Indekeu, J. Meunier, and E. Rolley. "Wetting and spreading," Rev. Mod. Phys. 81, 739-805 (2009)

23 J. H. Snoeijer and B. Andreotti, "Moving contact lines: scales, regimes, and dynamical transitions," Ann. Rev. Fluid Mech. 45, 269-292 (2013).

24 S. H. Smith, "On initial value problems for the flow in a thin sheet of viscous liquid," J. Appl. Math. Phys. 20, 556-560 (1969).

25 S. K. Wilson and B. R. Duffy, "Unidirectional flow of a thin rivulet on a vertical substrate subject to a prescribed uniform shear stress at its free surface," Phys. Fluids 17, 108105 (2005). 
26 J. M. Sullivan, S. K. Wilson, and B. R. Duffy, "A thin rivulet of perfectly wetting fluid subject to a longitudinal surface shear stress," Q. J. Mech. Appl. Math. 61, 25-61 (2008).

27 The solution has features in common with the similarity solution of Betelú and Diez ${ }^{21}$ in which two semi-infinite contact lines meet to form a semi-infinite "dry line" that vanishes at a "welding point" which moves at constant velocity; however, in the solution of Betelú and Diez ${ }^{21}$ surface-tension effects are dominant, whereas in the present solution they are neglected.

28 Alternatively one may obtain equation (59), which is the leading order form of (21) in the purely shear-driven limit $|\tau| \rightarrow \infty$, by rescaling (21) according to $\eta \rightarrow|\tau|^{-1 / 2} \eta$ and $c \rightarrow|\tau| c$ (with $F$ unscaled), and then taking the leading-order equation in the limit $|\tau| \rightarrow \infty$.

29 Y. M. Yatim, "Unsteady Flows of Thin Films," Ph. D. Thesis, University of Strathclyde, Glasgow, United Kingdom (2010).

30 Preliminary calculations suggest that there are solutions analogous to (19) describing a slender steadily translating dry patch in a thin fluid film in the case when surface-tension effects dominate over gravity in the transverse direction $\left(\epsilon^{2} \gg B\right)$; these remain an open topic for future work. 